\title{
HPLC in Natural Product Analysis: The Detection Issue
}

\author{
Author \\ Affiliation

Key words
high-performance liquid
chromatography
detection
hyphenation
natural products
dereplication
plant extracts

\author{
Jean-Luc Wolfender
}

Laboratory of Pharmacognosy and Phytochemistry, School of Pharmaceutical Sciences, University of Geneva, University of Lausanne, Geneva, Switzerland received July 31,2008

revised October 14, 2008

accepted October 23, 2008

\section{Bibliography}

Dol $10.1055 / \mathrm{s}-0028-1088393$

Planta Med 2009; 75: 719-734

(c) Georg Thieme Verlag KG

Stuttgart · New York

Published online January 14 ,

2009

ISSN 0032-0943

\section{Correspondence}

\section{Jean-Luc Wolfender}

Laboratory of Pharmacognosy

and Phytochemistry

School of Pharmaceutical

Sciences

University of Geneva

University of Lausanne

30 Quai Ernest-Ansermet

1211 Geneva 4

Switzerland

Tel.: +41-22-379-3385

Fax: +41-22-379-3399

jean-luc.wolfender@unige.ch

\section{Abstract}

$\nabla$

High-performance liquid chromatography (HPLC) is a very powerful and versatile chromatographic technique for the separation of natural products (NPs) in complex matrices, such as crude extracts for selective detection and quantification or general profiling. The method is widespread and has been adapted to the analysis of a broad range of NPs generally without the need for complex sample preparation. The choice of the appropriate detection method in HPLC is crucial because of the diversity of NPs and the fact that there is no single technique for their efficient detection. In this review both qualitative and quantitative applications of HPLC with UV, DAD, FD, ECD, RID, FID, CL, ESLD, CAD, MS, MS-MS, and NMR are covered to provide a general, rather than an exhaustive, overview. The potential and limitations as well as some new trends in HPLC hyphenation are discussed.

\section{Abbreviations \\ $\nabla$ \\ APCI: atmospheric pressure chemical ionisation \\ API: atmospheric pressure ionisation \\ APPI: atmospheric pressure photoionisation \\ CAD: charged aerosol detection \\ CapNMR: capillary NMR \\ CID: collision-induced dissociation \\ CL: chemiluminescence}

\section{Introduction \\ $\nabla$}

Natural products (NPs) have provided the inspiration for most of the active ingredients in medicines. The reason for this success in drug discovery can probably be explained by their high chemical diversity, the effects of evolutionary pressure to create biologically active molecules,
DAD: photodiode array detection

ECD: electrochemical detection

EI: electron impact

ELSD: evaporative light scattering detection

ESI: electrospray ionisation

FD: fluorescence detection

FID: $\quad$ flame ionisation detection

FT: $\quad$ Fourier transform

HCA: hierarchical clustering analysis

HPLC: high-performance liquid chromatography

HR: high resolution

LOD: limit of detection

LOQ: limit of quantification

LR: low resolution

MS: $\quad$ mass spectrometry

MS-MS: tandem mass spectrometry

$\mathrm{MS}^{\mathrm{n}}$ : multiple-stage mass spectrometry

MVDA: multivariate data analysis

NI: negative ion

NMR: nuclear magnetic resonance

PCA: principal component analysis

PI: positive ion

Q: $\quad$ quadrupole

QQQ: $\quad$ triple quadrupole

RID: refractive index detection

SPE: $\quad$ solid-phase extraction

TOF: time of flight

UHPLC: ultra high-pressure liquid chromatography

UV: ultraviolet 
tabolomics [2], which aims at measuring all of the metabolites in an organism qualitatively and quantitatively. The complex and convoluted nature of the crude extracts analysed (from plants, marine organisms, fermentation broths, etc.) also contributes to the difficulty of NP detection. Furthermore, the analytes can be present in large or very small amounts, and, according to the type of study (quantification, standardisation, fingerprinting, screening, trace analysis, etc.), very sensitive and selective methods may be needed for their detection.

The analysis of individual NPs in complex crude extracts requires efficient separation methods prior to their detection. In this respect, high-performance liquid chromatography (HPLC) has been recognised since the early $1980 \mathrm{~s}$ as the most versatile technique for the efficient separation of NPs directly in crude mixtures without the need for complex sample preparation [3]. HPLC has greatly developed through the years in terms of convenience, speed, choice of column stationary phases, high sensitivity, applicability to a broad variety of sample matrices, and ability to hyphenate the chromatographic method to spectroscopic detectors [4]. From the chromatography viewpoint, the development of columns with different phase chemistry (especially reversed phase) enabled the separation of almost any type of NPs. The latest developments of HPLC, including the recent introduction of very $\mathrm{pH}$-stable phases, sub-2- $\mu \mathrm{m}$ particles [5], and monolith columns, have considerably improved the performances of HPLC systems in terms of resolution, speed, and reproducibility. Efficiencies exceeding 100,000 plates and peak capacities over 900 can be attained by coupling columns together [6]. For the separation of crude extracts, either raw mixtures or samples enriched by extraction via simple SPE or LLE are injected. The separations are performed mostly in reversed-phase chromatography on C18 material with the $\mathrm{ACN}-\mathrm{H}_{2} \mathrm{O}$ or $\mathrm{MeOH}-\mathrm{H}_{2} \mathrm{O}$ solvent system in the gradient elution mode. In order to improve the separation efficiency, various modifiers are added to the mobile phase that might strongly influence the sensitivity of detection. In multiplehyphenated systems, the presence of several different detectors online (hypernated systems) leads to the need for an eluent composition that is compatible with all detectors [7].

HPLC is used extensively for NP profiling and fingerprinting, for quantitative analyses, and for quality control purposes. In this context, a brief overview of the methods available for NP detection is provided here. For each detector, the potential range of NPs to which they apply, their sensitivity, their selectivity, and their potential to provide online structure information will be discussed. A summary of the characteristic features of the main HPLC detectors is given in $\bullet$ Table 1.

Two main types of detectors can be defined: simple detectors used for the recording of chromatographic traces for profiling or quantification purposes (e.g., UV, ELSD, ECD) and detectors for hyphenated systems that generate multidimensional data (chromatographic and spectroscopic) for online identification and dereplication purposes (e.g., UV-DAD, MS, NMR).

\section{Detection Methods for Profiling and Quantification $\nabla$}

\section{HPLC-UV (ultraviolet detection)}

Among all HPLC detectors, the most simple and the most widely used is UV [8]. Although it suffers from some limitations, particularly for NPs that do not possess UV chromophores, it has the best combination of sensitivity, linearity, versatility, and reliability of all the LC detectors developed so far. Most NPs absorb UV light in the range of $200-550 \mathrm{~nm}$, including all substances having one or more double bonds and all substances that have unshared electrons. Thus, even compounds having weak chromophores, such as triterpene glycosides, can be successfully detected by UV at short wavelengths (203 nm) [9]. In this case, however, several mobile-phase constituents that exhibit high UV cut-offs should be avoided because they might blind the detection of NPs with weak chromophores [10].

In UV detection, the relationship between the intensity of light transmitted through the detector cell and the solute concentra-

Table 1 Performance of the different detectors for HPLC

\begin{tabular}{|c|c|c|c|c|c|c|c|c|c|}
\hline & Type & $\begin{array}{l}\text { Dynamic } \\
\text { range }\end{array}$ & Sensitivity & Specificity & $\begin{array}{l}\text { Chromato- } \\
\text { graphic } \\
\text { compa- } \\
\text { tibility }\end{array}$ & $\begin{array}{l}\text { Structural } \\
\text { information }\end{array}$ & $\begin{array}{l}\text { Ease } \\
\text { of use }\end{array}$ & $\begin{array}{l}\text { Wide- } \\
\text { spread } \\
\text { use for } \\
\text { NPs }\end{array}$ & Cost \\
\hline UV & Spec. & $* * *$ & $* * *$ & $* *$ & $* * * \mathrm{c}$ & * & $* * * *$ & $* * * *$ & $*$ \\
\hline $\mathrm{DAD}$ & Spec & $* * *$ & $* * *$ & $* *$ & $* * * c$ & $* *$ & $* * * *$ & $* * *$ & $* *$ \\
\hline $\mathrm{FD}$ & Spec & $* *$ & $* * * *$ & $* * *$ & $* * * *$ & $*$ & $* * *$ & $*$ & $* *$ \\
\hline EC & Spec & $* * * *$ & $* * * *$ & $* *$ & $* *$ & - & $* *$ & $* *$ & $*$ \\
\hline RID & Uni. & $* *$ & $* *$ & - & $* \mathrm{~d}, \mathrm{e}$ & - & $* * * *$ & $*$ & $*$ \\
\hline ELSD & Uni. & $* * a$ & $* *$ & - & $* * \mathrm{e}$ & - & $* * * *$ & $*$ & $*$ \\
\hline CAD & Uni. & $* * * * a$ & $* * *$ & - & $* * \mathrm{e}$ & - & $* * * *$ & -9 & $* *$ \\
\hline MS & $\begin{array}{l}\text { Uni./ } \\
\text { Spec. }\end{array}$ & $* *$ & $* * * *$ & $* * *$ & $* * \mathrm{e}$ & $* * *$ & $* *$ & $* * *$ & $* * *$ \\
\hline MS-MS & $\begin{array}{l}\text { Uni./ } \\
\text { Spec. }\end{array}$ & $* *$ & $* * * *$ & $* * * *$ & $* * \mathrm{e}$ & $* * * *$ & * & $* * *$ & $* * * *$ \\
\hline NMR & Uni. & $* * b$ & $*$ & $*$ & $* f$ & $* * * *$ & $*$ & $* *$ & $* * * *$ \\
\hline $\begin{array}{l}\text { Note: Un } \\
{ }^{\mathrm{a}} \text { Non-lin } \\
\text { b NMR pr } \\
{ }^{\mathrm{c}} \text { Require } \\
{ }^{\mathrm{d}} \text { Not con } \\
{ }^{\mathrm{e}} \text { Usually } \\
{ }^{\mathrm{f}} \text { Need de } \\
{ }^{\mathrm{g}} \text { Relative }\end{array}$ & $\begin{array}{l}\text { niversal; } \\
\text { esponse. } \\
\text { es absolu } \\
\text { Ivents an } \\
\text { ible with } \\
\text { compatit } \\
\text { rated wat } \\
\text { ew techn }\end{array}$ & $\begin{array}{l}\text { specific. } \\
\text { antification b } \\
\text { ers with low } ~ \\
\text { ents. } \\
\text { h non-volatil } \\
\text { d deuterated }\end{array}$ & $\begin{array}{l}\text { not used in thi } \\
\text { ut-offs. } \\
\text { tffers. } \\
\text { difiers in the on }\end{array}$ & $y$ in the on-flow & de. & & & & \\
\hline
\end{tabular}


tion is given by Beer's Law [11]. The two factors that control the detector sensitivity are the magnitude of the extinction coefficient of the analyte of interest at a given wavelength and the path length of the light passing through the UV cell. The sensitivity will increase with increasing path length, but a compromise must be found with the cell volume in order to avoid peak dispersion [8].

Three types of UV detectors are available: fixed wavelength, multiple wavelength, or photodiode array (DAD). The fixedwavelength detector is the least expensive and has higher intrinsic sensitivity because the light is emitted at specific wavelengths with given lamps. However, the multiple-wavelength detector is more versatile and can often compensate for its lower sensitivity when a wavelength with the highest extinction coefficient for the solutes of interest is chosen. The photodiode array will be treated in the hyphenation section, as it can provide complete UV spectra of the constituent during separation. Limits of detection (LODs) can reach $10^{-8} \mathrm{~g} / \mathrm{mL}$, with a linear dynamic range of about three orders of magnitude.

Often, the separation of crude extracts requires a gradient elution which cause a shift of the baseline at low wavelengths that can be avoided when working with modifiers having low UV cutoffs such as phosphate buffers or trifluoroacetic acid (TFA). These modifiers, however, are not optimal in multi-hyphenated systems with HPLC-MS (not volatile, ion suppression) and should be avoided [12].

Many publications refer to the application of HPLC-UV at a single wavelength for the detection of nearly all NPs having a chromophore for both profiling and quantification purposes. An exhaustive survey would exceed the frame of this paper. Many applications for flavonoids, terpenes, alkaloids, coumarins, alkamides, and polyacetylene have been summarised in a review [13]. Some selected quantitative methods are discussed here.

Being simple and inexpensive, HPLC-UV is used in several pharmacopoeias for the quantification of individual NPs in the quality control of herbal drugs or phytopreparations. In the U.S. and European pharmacopoeias [14], [15], HPLC-UV (203 nm) methods are used for the determination of the total ginsenoside content of Ginseng roots and the measurement of the ratio of the ginsenosides, $\mathrm{Rg} 1$ and $\mathrm{Rb} 1$ [16]. This analysis is indeed important to assess pharmacological effects, as a low $\mathrm{Rg} 1 / \mathrm{Rb} 1$ ratio is linked to the calming properties of American ginseng, while the high $\operatorname{Rg} 1 / \mathrm{Rb} 1$ ratio could be the reason for the stimulating characteristics of Asian ginseng. HPLC-UV also has been used for the quantification of flavonoid, naphthodianthrone, and phloroglucinol constituents of Hypericum perforatum. The developed method used a ternary solvent system in the gradient mode (50 min) and enabled a baseline separation of 16 main constituents, which were quantified based on their relative response factors at $270 \mathrm{~nm}$ and compared with rutin as an external standard [17]. A more rapid method (5 min) using fast-gradient elution for a specific determination of naphthodianthrones $(590 \mathrm{~nm})$ and phloroglucinols ( $270 \mathrm{~nm}$ ) also has been reported [18]. Many papers refer to the HPLC-UV analysis of another important herbal drug, Ginkgo biloba. A complete profiling of most of the flavonoids $(350 \mathrm{~nm}$ ) (33 compounds detected by comparison with standards) was reported by Hasler et al. [19]. The same authors also proposed an HPLC-UV method, which includes the hydrolysis of all glycosides and the subsequent quantification of three main flavonoid aglycones (quercetin, kaempferol, and isorhamnetin) for the determination of the total flavonoid content. Recently, another HPLC-UV method ( $350 \mathrm{~nm}$ ) has been reported, which includes the quantification of two glycosides (rutin and quercitrin) as markers, in addition to the three aglycones [20]. An analysis of 10 commercial samples revealed that problems of adulteration with rutin exist to fulfil the total flavonoid requirement (24\%), and thus in-depth quality control needs complete profiling [21]. Recently a multivariate data analysis (MVDA) of Ginkgo products was performed based on their HPLC-UV profiles, and the results were compared with the traditional flavonol quantification method [22]. This analysis revealed that there are significant variations in the relative concentrations of individual flavonols in commercial products. The other active ingredients in Ginkgo are the terpene trilactones, which are standardised at $6 \%$ in the extracts. These terpenes exhibit rather weak chromophores $(\lambda 220 \mathrm{~nm}$ ) and are difficult to detect in the presence of the strongly UV-absorbing flavonoids [23]. They have thus successfully been quantified by other methods such as ELSD [24] (see below). In Gingko, the level of highly allergenic alkylphenols (ginkgolic acids, cardanols, and cardols) also needs to be assessed, and a limit of $5 \mathrm{ppm}$ has been proposed in the EU and U.S. pharmacopoeias. An in-depth analysis requires GC-MS [25] or HPLC-MS [26], but their total amount can be determined by a simple HPLC-UV $(210 \mathrm{~nm})$ analysis without enrichment procedures after peak purity assessment by HPLC-DAD-MS [27].

In other cases, for the same plant extract, quantification may require both HPLC-UV and HPLC-MS. This was necessary for the standardisation of the constituents of woad (Isatis tinctoria). In this case, when possible, HPLC-UV was preferred to HPLC-MS because of the lower RSD obtained [28].

Recently, other simple HPLC-UV methods for the standardisation of new herbal supplements coming on the market such as Pueraria lobata, also known as Kudzu (Japan), have been developed. In this case, a fully validated method following the ICH guidelines for the quantification of the isoflavone content of this plant has been reported [29]. Similarly a method for profiling coumarins in different Angelica species has also been validated [30].

HPLC-UV methods also can be used to compare the fingerprint profiles of closely related species or the same species from different locations. This was the case for Herba Oldenlandiae, a plant of the Chinese pharmacopoeia, which can be adulterated by different Oldenlandia species. A comparative analysis of chemical components was obtained by software calculation of correlation coefficients of the entire UV chromatograms for appraising similarity [31]. Fingerprint-based similarity also can be employed for chemotaxonomic study by applying hierarchical clustering analysis (HCA) and principal component analysis (PCA) to a set of HPLC-UV profiles, as was performed for different Taxus species [32].

In $\odot$ Fig. 1, the profiling of the constituents of the crude extract of the model plant Arabidopsis thaliana obtained with UV detection at two standard wavelengths ( 254 and $350 \mathrm{~nm}$ ) is illustrated. A selective detection of flavonoids (F) and sinapoyl derivatives (S) was performed at $350 \mathrm{~nm}$. Detection at $254 \mathrm{~nm}$ was less selective as more UV active constituents were recorded; this included glucosinolates $(G)$, which have UV maxima at $227 \mathrm{~nm}$. However, fewer peaks were recorded with UV than with MS or ELSD detection methods (see below). In this example, the high chromatographic resolution was provided by ultra high-pressure liquid chromatography (UHPLC) [5], which yielded very high peak capacities (see inset $c$ in the MS trace) [33], [34]. 


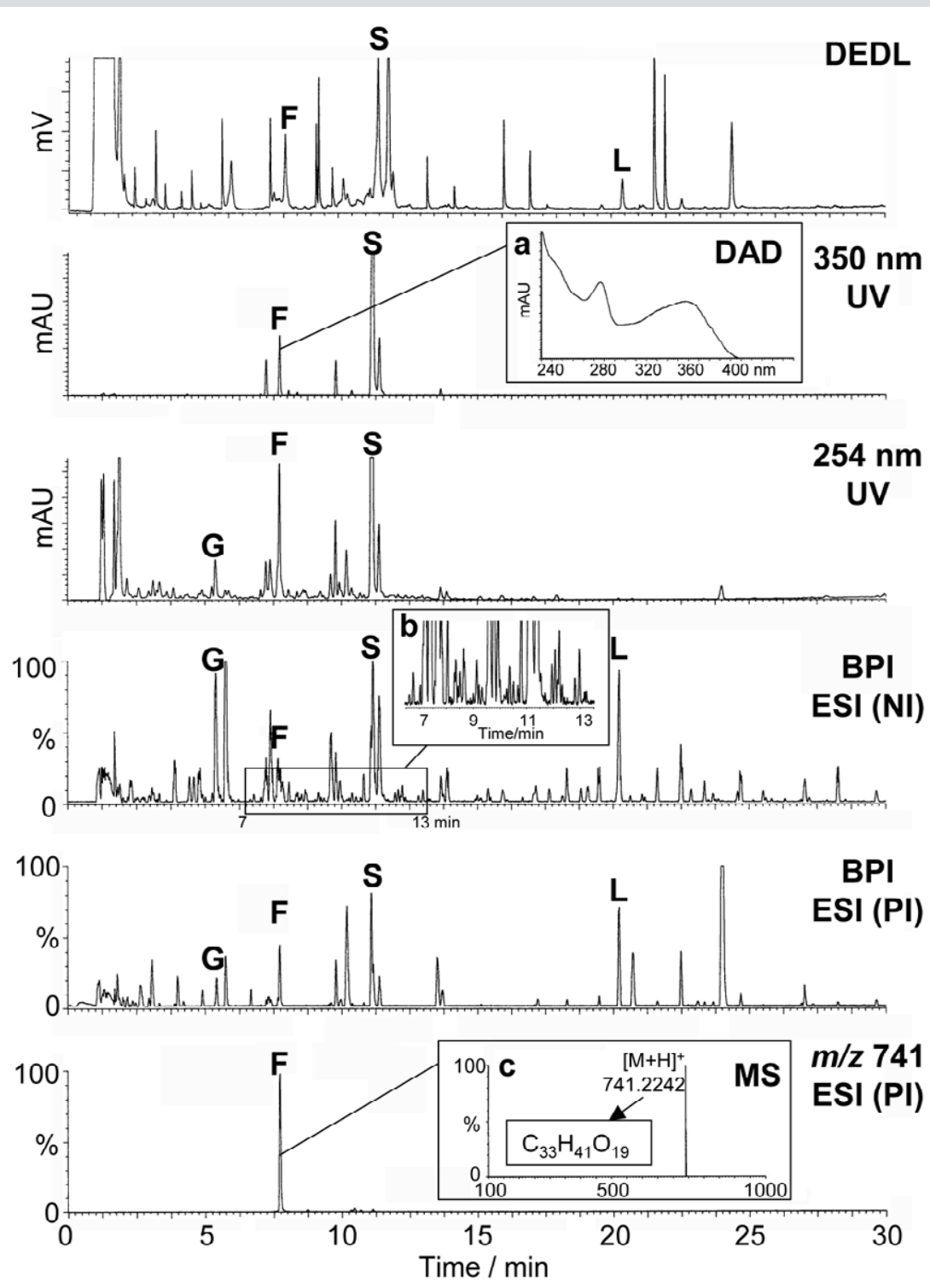

Fig. 1 Comparison of different detection techniques used to monitor the UPLC separation of the crude leaf extract of Arabidopsis thaliana. UPLC conditions: Column BEH C18:1.0×50 mm and $2.1 \times 150 \mathrm{~mm}, 1.7 \mu \mathrm{m}$ (Waters Acquity); gradient $5 \%$ to $98 \%$ ACN ( $0.1 \%$ formic acid) in 45 min; temperature $35^{\circ} \mathrm{C}$; flow rate $300 \mu \mathrm{L} / \mathrm{min}$. ESI-TOF-MS detection: LCT Premier (Waters); capillary voltage $2800 \mathrm{~V}$; cone voltage $40 \mathrm{~V}$; desolvation temperature $250^{\circ} \mathrm{C}$; desolvation gas flow $550 \mathrm{~L} / \mathrm{h}$; $\mathrm{PI}$ and $\mathrm{NI}$ in the range $m / z=100-1000$ with a scan time of 0.25 s. UV-DAD detection: $220-500 \mathrm{~nm}$. DEDL detection: SEDERE sedex85, $P_{\text {neb }}=3 \mathrm{bar}, T_{\text {evap }}=50^{\circ} \mathrm{C}$, gain 8 . Compound $F$ is a flavonol glycoside, $G$ a glucosinolate, $\mathrm{S}$ a synapoyl derivative, and $\mathrm{L}$ a galactolipid. Insets a and c: UV-DAD and TOF-MS spectra of F. Inset b: Zoom into the BPI-ESI NI chromatogram showing the important number of minor peaks detected.

\section{HPLC-FD (fluorescence detection)}

Compared with HPLC-UV, fluorescence detection (FD) affords much greater sensitivity and selectivity ( $\bullet$ Table 1 ). In fluorescence, the molecular absorption of a photon triggers the emission of another photon with a longer wavelength. This difference in wavelengths (absorption $v s$. emission) provides more selectivity, and the fluorescent light is measured against a very low-light background, thus improving the $\mathrm{S} / \mathrm{N}$ ratio. Unfortunately, relatively few NPs fluoresce in a practical range of wavelengths. However, many substances can be made to fluoresce by forming appropriate derivatives [8].

Compared with UV, FD has been scarcely used for the detection NPs. Most of the applications are related to the very sensitive detection of aflatoxins in food because this class of NPs contains natural fluorescence [35]. An FD method for the quantification of aflatoxins in herbs also has been recently reported [36]. It combines sample cleanup with immunoaffinity columns followed by HPLC-FD, and it enabled the detection of aflatoxin content ranging from 7 to $20 \mu \mathrm{g} / \mathrm{kg}$ in the plant material. HPLC-FD also has been used for the screening of the major benzophenanthridine alkaloids produced by cell cultures of Eschscholtzia californica [37]. Thanks to the selectivity of the method (excitation $330 \mathrm{~nm}$, emission $570 \mathrm{~nm}$ ), the sample preparation was notably simplified compared with previous methods. The detection of NPs that do not naturally fluoresce has been successfully achieved after the addition of fluorescent tags. This was the case for the specific and sensitive detection of the plant hormone jasmonic acid (LOD $3 \mathrm{ng} / \mathrm{mL}$ ), which was tagged with the ADAM reagent [38].

\section{HPLC-CL (chemiluminescence)}

Similar to fluorescence, chemiluminescence can be defined as the emission of light from a molecule or atom in an electronically excited state produced by a chemical reaction at an ordinary temperature without any associated generation of heat. Chemiluminescent $(\mathrm{CL})$ nitrogen detection is a relatively new technology for the detection of nitrogen-containing molecules, including a broad range of pharmaceuticals with very high sensitivity up to the femtogram range [39]. Because many compounds are not chemiluminescent, they can only be detected chemiluminescently after being derivatised. The development of chemiluminogenic reagents has helped to extend the application of $\mathrm{CL}$ 
detection, especially for pharmaceutical biomedical applications [39]. In applications with HPLC, the reagents are applied postcolumn. For example, the addition of potassium hexacyanoferrate(III) enables the detection of various carboxylic acids in the form of $\mathrm{N}$-(4-aminobutyl)- $\mathrm{N}$-ethylisoluminol derivatives [40]. While being very sensitive, the method so far has been very scarcely applied to the analysis of crude plant extracts. A study reported the sensitive HPLC-CL detection of flavonoids (LOD $3 \mathrm{ng} / \mathrm{mL}$ ) in phytopharmaceuticals containing Hippophae rhamnoides. The procedure was based on chemiluminescent enhancement by flavonols of the cerium(IV)-rhodamine 6G system in a sulphuric acid medium [41].

\section{HPLC-ECD (electrochemical detection)}

Many NPs have electroactive groups and are readily measurable and detectable by LC with electrochemical detection (ECD). This detection method is inexpensive, sensitive, selective, and widely accepted ( $\odot$ Table 1 ) [10]. It is less selective than FD; however, NPs with electrochemical activity are common, and the technique can be applied to a large number of analytes in either the oxidation or reduction modes. Numerous functional groups are sensitive to oxidation, including phenols, aromatics, amines, thiols, and quinolines, and many are compatible with reduction, such as olefins, esters, ketones, aldehydes, ethers, and quinones. ECD differs from other methods of detection because it alters the sample. Cells for HPLC-ECD usually consist of three electrodes, the working, counter, and reference electrodes, which can be aligned in several different geometries, although two main designs exist. In coulometric systems, the eluent is directed through the electrode, while in amperometric systems, the eluent passes over it. ECD detection is usually performed by maintaining the potential of the working electrode at a fixed value relative to the potential of the electrolyte, which is measured by the reference electrode. The fixed potential difference applied between the working and the reference electrodes drives the electrochemical reaction, and the resulting current is measured as a function of the elution time. This allows for detection limits at the pmol level. The selectivity of ECD depends on the accessible potential range, the number of compounds that are active in this range, and the half-widths of the individual signals [10]. Pulsed techniques, which use multi-step potential-time waveforms to realise amperometric/coulometric detection while maintaining uniform and reproducible electrode activity, are also available for detection [42].

Modern interest in HPLC-ECD is stimulated by the recognition that electrochemistry is ideal for the study of the metabolism of aromatic compounds. However, proper use of LC-ECD requires knowledge of redox reactions and their dependence on mobilephase composition. In this respect, the study of electrode reaction mechanisms can sometimes aid the elucidation of the mechanism of their interaction with living cells, such as for antioxidant NPs [43]. The interest in the method resides also in the fact that the ECD can be easily miniaturised and used in fully integrated chip-based systems.

HPLC-ECD is commonly used in many pharmaceutical, clinical, and biochemical laboratories [10], [44]. Because antioxidants are electrochemically active, HPLC-ECD is well suited for their accurate quantification. In this respect, the method has permitted the detection and quantification of very low levels of antioxidants, such as carnosic acid in food ingredients [45]. In plant extract profiling, coulometric electrode array detection has allowed for the fingerprinting of 25 constituents (polyphenols and tanshinones) in Salvia miltiorrhiza after a careful selection of the optimal mobile-phase $\mathrm{pH}$ and working potentials [46]. The successful detection of various small phenolics, such as coumaric or ferulic acids, with a gold polycrystalline electrode has been reported recently [47]. This method was also used for the quantification of the vanilloid content in Capsicum annuum extracts [48]. ECD was found to be more sensitive than UV for the normal-phase HPLC profiling of diarylheptanoids in Alpinia officinarum (lesser galangal) [49]. Similarly, the simultaneous determination of hyperforin and total hypericin in Hypericum perforatum was achieved after the photoconversion of the protoforms of hypericins in extracts and tablets [50].

The antioxidant potential of polyphenols can be estimated by HPLC with coulometric array detection because they are reducing agents, and their electrochemical responses result from the donation of electrons. Some degree of correlation between the electrochemical profiles of 17 flavonoids and 3 cinnamic acid derivatives and 3 different antioxidant assays was established [52]. HPLC-ECD also has been used in many applications related to the analysis of drugs in body fluids [44], [10]. Artemisinin, for example, was selectively detected and quantified in plasma using a glassy carbon electrode by reductive ECD [52]. Similarly, acteoside was analysed by amperometric detection in rat blood and brain microdialysate in a pharmacokinetic study [53]. Recently, different amino acid derivatives characteristic for Korean ginseng were analysed by pulsed amperometric detection in both ginseng and plasma samples [54].

\section{HPLC-RID (refractive index detection)}

The detectors described previously are selective for analytes having specific spectroscopic or physicochemical properties. In order to obtain a more universal response, other detectors have been developed.

The first, most simple, and least expensive of these detectors is the refractive index detector (RID), which was reported by Tiselius and Claesson [55] in 1942. It was indeed the first effective inline LC detector. However, RID lacks sensitivity, is very susceptible to changes in ambient temperature, pressure, and flow rate, and cannot be used for gradient elution. Despite these disadvantages, this detector has been useful for detecting compounds such as carbohydrates and polymers.

Recent applications in the NP field are very scarce, although this method was used for the quantification of sugars in strawberry fruits [56]. HPLC-RID was also used to determine the sugar concentration in different dry extracts of Hypericum perforatum after extensive SPE cleanup [57]. More recently, the terpene trilactones of Ginkgo biloba were successfully quantified by HPLC-RID after a very specific sample preparation based on the ionisation of these terpenes (RSD $<3 \%$, LOD $0.1 \mu \mathrm{g}$, linear range $0.1-12 \mu \mathrm{g}$ ) [58].

Because of its inherent limitations, HPLC-RID has been favourably replaced by other universal detection methods such as those described below.

\section{HPLC-ELSD (evaporative light-scattering detection)}

Evaporative light-scattering detection (ELSD) was introduced in 1966 by Ford and Kennard [59]. ELSD is a quasi-universal detector for liquid chromatography, as it can detect any analyte less volatile than the mobile phase, regardless of the optical, electrochemical, or other analyte properties [60]. In this detector, the eluent is nebulised using a flow of nitrogen, and the resulting aerosol is transported through a heated drift tube where the vol- 
atile components and solvents are evaporated. The remaining solid fraction is subsequently introduced into a detection cell where a light beam is directed onto the particles that will cause scattering of the incident light detected by a photodiode or a photomultiplier. The most important parameters affecting the ELSD signal response are the nebuliser gas flow rate and the drift tube temperature [61]. The gas flow rate influences the droplet size of the column effluent before evaporation occurs. Higher flow rates result in the formation of smaller aerosol droplets and less scattering of light, with subsequent lower sensitivity, but they allow for a more stable baseline. The drift tube temperature facilitates the evaporation of the nebulised aerosol so that the light-scattering response of the non-volatile solute can exclusively be determined. Higher temperatures are needed for the mobile phase of high-aqueous-content or non-volatile solutes versus semi-volatile ones. However, the new generation of ELSD is able to vaporise the eluent at low temperature, facilitating the detection of semi-volatile analytes. It is pertinent to optimise these parameters to ensure that the optimal signal to noise ratio $(\mathrm{S} / \mathrm{N})$ is achieved [62]. ELSD is a mass-dependent detector, in contrast to UV, which is a concentration-dependent detector, and the generated response does not depend on the spectral or physicochemical properties of the analyte. In theory, this means that ELSD generates a similar response for equal amounts of mass present and thus a universal response factor. However, the ELSD response also depends on the volatility of the compound and the mobile-phase composition (in the case of gradient elution), which makes such quantification procedures experimentally inaccessible. Thus, this interplay of several factors leads to a non-linear response, which is one disadvantage of this type of detector and ultimately renders direct linear regression for making calibration curves inaccurate [63]. In order to avoid the variation generated by the gradient elution, the post-column addition of mobile-phase flow having an opposite composition is an interesting alternative [64].

The increased interest in ELSD as a universal detector is that it is more compatible with gradient elution than RID, and it is much less costly and easier to maintain than an HPLC-MS platform (see below). However, it is used mostly as an additional detector rather than a substitute. Recent papers have discussed advantages and potentialities as well as several limitations of the method for both NP and pharmaceutical applications [60], [63], [65].

In applications related to NPs, ESLD has been used mainly for the detection of compounds with weak chromophores such as terpenes, in both aglycone [62] and glycosidic forms [66], saponins [67], and some alkaloids [68]. The method also has been applied in complement to HPLC-MS for surveying NP libraries [69]. In this case, a wide range of products, including UV-active phenolics, were screened, and an ELSD signal was obtained for all compounds ( $>100 \mathrm{ng}$ injected). ELSD has been used for the quantitative determination of the relevant active principles of herbal drugs. A recent method for the detection of 14 ginsenosides in Korean red ginseng, using digoxin as an internal standard, has been validated and applied to several ginseng products [67]. All calibration curves showed good linearity using a log-log transformation $\left(R^{2}>0.9833\right)$, and LODs of $0.075-0.30 \mu \mathrm{g}$ and LOQs of $0.125-$ $0.80 \mu \mathrm{g}$ were achieved. Thus, ELSD represents an interesting simple alternative to the UV detection method (203 nm) of the pharmacopoeia. However, both UV and ELSD detections are often necessary for profiling extracts, as was shown for the simultaneous determination of triterpenoids (ELSD) and phytoecdysones (UV) in roots of Achyranthes bidentata (Amaranthaceae) [70].
The weakly UV-active ginkgolide and bilobalide of Ginkgo were also efficiently quantified by ELSD [71], [62] Camponovo et al. [71] noticed a specific response of ELSD for terpene trilactones versus flavonoids when UV detection ( $220 \mathrm{~nm}$ ) and ELSD detection were compared. Dubber et al. [62] were able to obtain a baseline separation of these compounds within $14 \mathrm{~min}$, and they have analysed several Ginkgo biloba products with LOQs of ca. $60 \mathrm{ng}$. Inter-day percentage standard deviations were all better than $6 \%$. ELSD thus represents an interesting alternative to the GC dosage usually performed for the standardisation of the terpene content of Ginkgo [72].

In the case of the crude extract profiling of Arabidopsis thaliana, shown in $\bullet$ Fig. 1, the ELSD response provided many more peaks than those detected in UV ( 254 and $350 \mathrm{~nm}$ ), especially for the detection of non-polar compounds (mainly lipids). Compounds detected by ELSD in this leaf extract included the notably different galactolipids such as (L), which were not visible in the UV traces.

\section{HPLC-CAD (charged aerosol detection)}

Charged aerosol detection (CAD) is a new technology for universal HPLC detection, which derived from ELSD and was introduced by Dison and Peterson in 2002 [73]. The first steps of the operating principle of $\mathrm{CAD}$ are roughly comparable to that of ELSD. Contrary to ELSD, however, with CAD, the dried particle stream is charged with a corona discharge needle, and the resulting electrical charge is measured by an electrometer [63]. The use of a corona discharge indicates some commonality with APCI-MS (see below); however, CAD operates by detecting charge particles with a selected mobility range rather than measuring individual gas-phase ions as in MS. Generally, CAD is more sensitive than ELSD ( Table 1 ), but this difference is strongly influenced by the LC modifier, as ELSD seems less sensitive to increases in salt concentration [63]. This detection method provides a uniform response to non-volatile analytes independently of their nature and a dynamic range up to 4 orders of magnitude [64].

For the detection of NPs with strong chromophores, UV usually performs better than CAD or ELSD. However, these latter detection methods are more dedicated to the analysis of non-UV-absorbing compounds, and of course their performances are in this case much better [63].

Because it is a new detection method, CAD has not yet been used for NP detection in crude extracts to our knowledge, although it has been used for a wide range of pharmaceutical products. Both CAD and ELSD were evaluated for a library of more than 700 compounds in the 150 to 700 Da range and failed only in the detection of two compounds having small MWs [74]. It is noteworthy that successful quantitative analysis of triacylglycerols (TGs) in plant oils has been achieved [75]. The method is advantageous in that no normalisation of the peak area is needed, as is the case in HPLC-MS, where large differences in responses can occur.

\section{HPLC-FID (flame ionisation detection)}

While considering a universal detection method for HPLC, it is worth mentioning that FID, which is a general detector for GC because it detects all carbon-containing molecules, also has been applied to HPLC. However, in both normal- and reversedphase modes, the LC mobile phases contain organic molecules that will blind the FID [76]. Thus, HPLC-FID coupling implies working with $100 \%$ water mobile phases. A major problem is that water is a very weak mobile phase in RPLC at room temperature. However, for selected compounds and with appropriate 
stationary phases, separations were realised with pure water at ambient temperature [77]. Another alternative is to work with superheated water in the HTLC mode because, when heated under pressure to temperatures between $80^{\circ} \mathrm{C}$ and $250{ }^{\circ} \mathrm{C}$, the polarity of liquid water decreases markedly to the extent that it can replace the organic modifier and act as the sole eluent [78].

While successful applications of HTLC-FID have been described [79] mainly for the separations alcohols, carbohydrates, and amino acids, the method has been used very rarely.

\section{HPLC-MS (mass spectrometry)}

The application of HPLC-MS in the analysis and characterisation of natural products has been recognised as a major breakthrough [80]. Indeed, while it is expensive, the use of a mass spectrometer as an HPLC detector provides excellent sensitivity and selectivity for the analysis of NPs in complex biological matrices. Furthermore, MS detection provides important structural information online such as MW, molecular formula, and diagnostic fragments, which are crucial for dereplication and rapid online characterisation of NPs (see below).

Historically, a main challenge in HPLC-MS was that high liquid flows from HPLC were not compatible with the high vacuum required for the mass spectrometer. Since the early 1980 s, a number of different interfaces have been developed to address this issue and overcome these difficulties [81], [82]. One obvious way to prevent the harmful effects of the LC mobile phase for the mass spectrometer was to remove the solvent prior to ionisation. This has led to the development of moving belt interfaces for sample introduction into the low-pressure plasma sources. The advantage of such devices was the possibility to generate electron impact (EI) spectra, for example, for flavonoids [83], but they suffered from many other disadvantages and were abandoned. Another more modern "transport-type" interface in LC-MS is the particle beam [84], but it has scarcely been applied to natural products analysis. A new generation of direct-EI interfaces coupled to nano-HPLC seems promising for generating such information-rich types of spectra [85].

The overwhelming popularity of HPLC-MS today is largely due to atmospheric pressure ionisation (API) interfaces including electrospray ionisation (ESI) and atmospheric pressure chemical ionisation (APCI). For both ESI and APCI, ionisation occurs at atmospheric pressure. Some combination of high voltage and heat is used to provide the ionisation that is needed to produce the ions that are assayed by the MS system. In ESI, the high-voltage field $(3-5 \mathrm{kV})$ produces nebulisation of the column effluent resulting in charged droplets that are directed toward the mass analyser. These droplets get smaller as they approach the entrance to the mass analyser. As the droplets get smaller, individual ions emerge in a process referred to as "ion evaporation"; these ions are then separated by the MS system. In APCI, heat is used to vaporise the column eluent, and then a corona discharge is used to ionise solvent molecules, which then produce the analyte ions via chemical ionisation mechanisms. More recently, a third type of ionisation source, termed atmospheric pressure photoionisation (APPI), has become available. In APPI, heat is used to vaporise the column eluent ( similar to APCI), but the ionisation is produced by way of an ultraviolet (UV) lamp that produces $10-\mathrm{eV}$ photons. Depending upon the solvent system used, the $10-\mathrm{eV}$ photons will ionise either the mobile-phase solvent or a dopant (e. g., toluene) added to the column effluent; these ions then produce the analyte ions through various ionisation mechanisms including charge or proton transfer [86].
These API methods generally provide a soft ionisation and mainly molecular ion species in the form of either protonated molecules $[\mathrm{M}+\mathrm{H}]^{+}$(positive-ion mode, PI) or deprotonated molecules $[\mathrm{M}-\mathrm{H}]^{-}$(negative-ion mode, NI). Different adducts (e.g., $[\mathrm{M}+\mathrm{Na}]^{+}(\mathrm{PI})$ or $\left[\mathrm{M}+\mathrm{HCOO}^{-}(\mathrm{NI})\right)$ are also produced, depending on the solutes and the modifiers used. It is important to understand that analyte ionisation is largely compound dependant and is governed mainly by proton affinity. As a rule of thumb for a good approximation, acidic molecules (e.g., carboxylic acids or phenolics) will produce mainly $[\mathrm{M}-\mathrm{H}]^{-}$in NI, while bases (e.g., alkaloids, amines) will generate $[\mathrm{M}+\mathrm{H}]^{+}$in PI. Compounds such as glycosides will have a high affinity for salts and will tend to form sodium adducts in PI. Molecule fragmentation or partial declustering of the adducts can be induced in one of the high-pressure regions of the ion beam from the source to the mass analyser by collision-induced dissociation (CID) either at the API source (in-source CID) or in conjunction with tandem MS (MS-MS) experiments [82].

For HPLC-MS applications, many types of mass spectrometers can be used. Those that are low resolution (LR), such as the single quadrupole $(\mathrm{Q})$ mass spectrometers, are the most commonly used and least expensive. Those giving high resolution (HR) and exact mass capabilities, such as time-of-flight (TOF) instruments, are becoming increasingly popular because they provide molecular formula information online and very precise ion trace extraction (+/- 0.01 DA) for a specific detection. For measuring structurally relevant fragments or for very specific detection, triple-quadrupole (QQQ) MS-MS systems are the most commonly used. They are particularly useful for bioanalytical assays and for specific quantification purposes in complex matrices through multiple reaction monitoring (MRM) [87]. Ion-trap mass spectrometers have the unique capability of producing multiplestage MS-MS $\left(\mathrm{MS}^{\mathrm{n}}\right)$ data that may be essential for structural elucidation purposes. In addition to these four kinds of mass spectrometers, there is a growing number of additional types, including hybrid systems that combine LR and HR analysers for specific applications [86], [81].

Besides their widespread use for qualitative analysis [dereplication studies and crude extract profiling (see below)], HPLC-MS and HPLC-MS-MS have been successfully used for the specific detection and quantification of a large range of natural products. While mainly simple quadrupole or triple-quadrupole analysers are considered for quantitative studies, TOF instruments have recently increasingly been used as quantification tools, even though historically they suffered from a narrow dynamic range [88].

A systematic HPLC-MS-ELSD study was conducted on 96 structurally diverse NPs, including phenolics, alkaloids, amides, terpenoids, steroids, and polyketides, with a short HPLC generic gradient (C18, 5-95\% ACN: $\mathrm{H}_{2} \mathrm{O} 0.1 \%$ formic acid in $10 \mathrm{~min}$ ). A good overall ionisation of most compounds with ESI in both PI and NI mode was obtained [69]. The generic separation used was suitable for the elution of 86 of these 96 compounds (RT > $1 \mathrm{~min}<10 \mathrm{~min}$ ); 75 were well detected in PI and 57 in NI, while all compounds were detected by ELSD ( $4 \mu \mathrm{g}$ on-column). A relatively universal detection can thus be obtained if both PI mode and NI mode are used or alternated during acquisition (see, for example, the profiling of $A$. thaliana constituents in Fig. 1). ESI (PI) is used mainly for alkaloids, APCI (PI) for pigments and carotenoids, ESI (PI/NI) for triterpene glycosides, and APCI or $\mathrm{ESI}(\mathrm{PI} / \mathrm{NI})$ for phenolics and flavonoids. APCI is generally more suitable for non-polar constituents, while ESI provides a softer 
ionisation and gives a good response for polar metabolites. On the other hand, $\mathrm{APCI}$ is generally recognised to be less prone to matrix effects than ESI [89]. Only a few studies have referred to the use of APPI for NP detection [90]. An interesting work on the rapid analysis of stilbenes and derivatives from downy mildewinfected grapevine has shown that APPI NI with acetone as doping reagent was more sensitive than ESI and provided cleaner spectra [91]. Another study on anthocyanidins in wines has shown that both APPI and ESI-MS allow the identification of a great number of these pigments. However, APPI ionisation was found to be more specific towards the analyte, and the background noise was lower [92]. In drug discovery, the performances of APPI, APCI, and ESI were evaluated on 106 standards and 241 proprietary drug candidates occupying a wide chemical space [93]. The results indicated that the detection rates in PI were $94 \%$ for APPI, $84 \%$ for APCI, and $84 \%$ for ESI. When PI and NI mode detection were combined, APPI detected $98 \%$ of the compounds, while APCI and ESI each detected $91 \%$. In APPI, for analytes of low polarity and low proton affinity, $\mathrm{M}^{+}$was the predominant ion species generated, while for high-polarity and high-proton affinity compounds, $[\mathrm{M}+\mathrm{H}]^{+}$ion species dominated the spectra. APPI is thus regarded by some authors [90] as a more universal detection method for HPLC-MS than APCI and ESI, but, at present, it is still not widely used in natural products analysis.

Because ionisation is compound dependant in HPLC-MS, the choice of the interface, the ion mode polarity, and the eluent composition might significantly affect the sensitivity. The case of the analysis the triterpene glycosides of ginseng (ginsenosides) is treated here to show how ionisation conditions were selected in different studies, as more than 30 papers have dealt with their HPLC-MS detection in either plant extracts formulation or body fluids. Indeed, while HPLC-UV [94], [95] and HPLCELSD [96] methods have been used for the detection of these saponins (see above), HPLC-MS is the method of choice. The latter provides sensitive and selective detection in shorter analysis time, as only isomers need to be separated and MS provides useful structural information for a confirmation of the identity of these glycosides.

ESI (NI) was used for qualitative [97] and quantitative [98] studies mainly on various Panax spp. extracts. In conjunction with multiple-stage MS-MS (MS ${ }^{\mathrm{n}}$ ) experiments, fragmentation of the $[\mathrm{M}-\mathrm{H}]^{-}$provided the identification of 25 ginsenosides [97]. Another study demonstrated that different negative-ion source conditions were necessary for an optimum ionisation of malonylated and non-malonylated ginsenosides [99]. Quantification of the ginsenosides Re, Rb1, and Rc in different dosage forms of ginseng was provided by integration of their specific $[\mathrm{M}-\mathrm{H}]^{-}$ion traces [98]. ESI (PI) was also used successfully for the detection and quantification of these triterpene glycosides. Wang et al. reported a quantification method in MS-MS in the single-reaction monitoring mode (SRM) for a very selective dosage of Rb1, Rc, and $\operatorname{Re}[100]$. In this case, both $[\mathrm{M}+\mathrm{Na}]^{+}$and $[\mathrm{M}+\mathrm{H}]^{+}$ions were observed, but the CID MS-MS spectra of the $[\mathrm{M}+\mathrm{H}]^{+}$species were found to be more informative than those of the sodiated adducts. ESI (PI) was also used more recently in other quantification studies of Panax ginseng [101] and root and leaves of Panax quinquefolium [102], with an RSD of about $8 \%$ in the latter case. With the same ionisation mode, a newly developed UHPLCQTOF-MS method has been efficiently used for the metabolite profiling of different Panax species that were efficiently differentiated after PCA of the data [103]. Recently, APCI (PI and NI mode) was also investigated, and 30 ginsenosides were determined. The NI mode provided $[\mathrm{M}-\mathrm{H}]^{-}$ions, while in the PI mode, characteristic thermal degradation ions were recorded [104]. Yu et al. also used APCI (NI) for the quantification of 6 ginsenosides as well as other products in a complex traditional Chinese medicine (TCM) preparation (Naodesheng injection) containing five plants, including Rhizoma Notoginseng [105]. In this case, the addition of $0.1 \%$ formic acid into the mobile phase ensured the formation of stable $\left[\mathrm{M}+\mathrm{HCOO}^{-}\right.$molecular ions. The detection of these ions in the SIM mode produced data with acceptable linearity, repeatability, precision and accuracy (LOQs $0.02-0.2 \mu \mathrm{g}$, linearity coefficient $>0.9900, \mathrm{RSD}<3 \%$ ). It is also noteworthy to mention that ginsenosides have been analysed and quantified in body fluids with similar HPLC-MS methods [106]. Thus, as shown here, depending on the conditions and the matrix studies, both APCI and ESI (PI and NI) enable the quantification and identification of ginsenoside provided that a correct optimisation is insured. Other types of saponins could also be well detected by LC-MS. This was the case, for example, for the evaluation of the steroidal saponin content of Rhizoma Paridis and Chinese multi-herb remedies that contain this plant [107].

Another well-studied plant is Ginkgo biloba, for which both the terpene (ginkgolides and bilobalide) and the flavonoid contents need to be quantified for standardisation purposes. Different HPLC-MS methods have been used for the quantification of the terpenes because their detection in UV is compromised by the strongly UV-absorbing flavonoids (see above). APCI (NI) enabled a direct analysis of several Ginkgo preparations without pre-purification and provided LODs in the picogram range. Because the detection relies on the specific extraction of the $[\mathrm{M}-\mathrm{H}]^{-}$, a fast HPLC gradient was applied (14 min), which provided a highthroughput survey of many samples [23]. More recently, ESI (NI) in MRM mode enabled an even more sensitive and specific detection by the monitoring of specific MS-MS transitions of these terpenoids from their precursor [ $\mathrm{M}-\mathrm{H}]^{-}$ions [108]. Ginkgolides have also been successfully analysed by APCI (NI) in MRM mode in plasma samples for pharmacokinetic studies [109]. Another validated method for the specific detection of these compounds relies on GC-MS [72]; in this case, however, a tedious sample preparation is needed.

Because of its high sensitivity and selectivity, HPLC-MS also represents a key technique for the detection of toxic compounds present at trace levels. For example, HPLC-MS-MS was evaluated for the quantification of urushiol congeners, which are responsible for poison oak dermatitis. ESI (NI) in the MRM mode yielded LODs in the low ppm range that are relevant for toxicological, clinical, and environmental studies of these allergens [110]. Similarly, pyrrolizidine alkaloids, a class of hepatotoxic and tumorigenic phytochemicals, have been analysed in honey samples and in extracts of Echium plantagineum. ESI (PI) provided good detection limits for these compounds; however, previous sample enrichment with strong cation-exchange SPE was mandatory [110]. The consumption of slimming regimens made of Chinese herbal preparations has caused intoxications associated with species of the Aristolochia genus, which contain highly nephrotoxic and carcinogenic metabolites called aristolochic acids. Their detection in commercial dietary supplements was successfully achieved in suspect preparations by using both UV and MS detection [112]. A recent LC-MS-MS method enabled the simultaneous detection of aristolochic acid I and the marker compounds fanghinoline and tetrandrine in many TCM preparations [113]. 
In $\odot$ Fig. 1, the type of detection provided by ESI in both NI and PI modes for the profiling of Arabidopsis thaliana constituents is compared with the detection obtained by the UV and ELSD methods. As shown, very complex MS base peak ion chromatograms (BPIs) were obtained in both PI and NI mode. The number of constituents detected was higher than that observed in UV and ELSD traces. It is noteworthy to observe that PI and NI modes do not generate similar chromatograms and that some of the compounds were detected in only one of the ionisation modes. This demonstrates that MS represents a rather universal detection method of crude extract profiling if both PI and NI mode are used complementarily. On the other hand, very specific detection can be obtained if the extracted chromatogram of a given ion is displayed. For example, the ion trace $m / z=741.22$ has been selected in $\bullet$ Fig. 1. This ion corresponds to the protonated molecule of a flavonoid triglycoside $\mathrm{F}$ having the molecular formula $\mathrm{C}_{33} \mathrm{H}_{41} \mathrm{O}_{19}$. Because TOF was used for detection in this example, a precise mass measurement was obtained (741.2242 Da), and the corresponding ion chromatogram was extracted in a narrow mass window of $0.01 \mathrm{Da}$. This enabled the very specific detection of this compound among the other constituents, which would ensure its successful quantification.

As shown, HPLC-MS represents a very versatile HPLC detection method. Most of the API interfaces available cover ionisation of the vast majority of NPs, provided that correct conditions are used. Of course, the applications to NPs are manifold, and more details can be found in dedicated reviews [80], [82], [114], [115]. Concerning the detection of NPs in biological fluids, an interesting review covers the aspects related to sample preparation and quantification of flavonoids, alkaloids, saponins, and sesquiterpenoids [116].

\section{HPLC-NMR (nuclear magnetic resonance)}

HPLC-NMR is used mainly for providing structural information online (see below), but, as ${ }^{1} \mathrm{H}-\mathrm{NMR}$ can detect all analyte-bearing protons, HPLC-NMR also can be regarded as a universal detection method [117]. However, it is not used for this purpose, mainly because of its lack of sensitivity and the high costs associated with the use of this technique ( $\bullet$ Table 1 ). HPLC- ${ }^{19}$ F-NMR has been used for the specific detection of fluorinated derivatives. This was the case for studying the metabolic fate of 3chloro-4-fluoroaniline in rat body fluids [118].

\section{Detection Methods for Online Structural Identification \\ $\nabla$}

Some of the spectroscopic methods used for the detection of NPs (DAD, MS, NMR, CD, IR) also can be used in hyphenation with HPLC to generate structurally relevant data for their online identification and dereplication purposes. When both chromatographic and spectroscopic dimensions are used, the methods are referred to as "LC-hyphenated techniques." Dereplication of NPs with these methods is strategic for guiding an efficient lead-finding process [119]. However, this task is not easy to perform, because no exhaustive LC spectroscopic databases are available. Furthermore, even if libraries of NP spectra could be efficiently generated on a given LC-hyphenated system, their widespread use is complicated by the fact that, contrary to GCMS, most of the data, especially HPLC-MS and HPLC-MS-MS, are instrument dependant [120]. Thus, for each new compound, both the order of the atoms and the stereochemical orientations have to be elucidated de novo. Consequently, a single LC-hyphenated technique does not exist that is capable of identifying online all secondary metabolites in a plant extract [121]. Ideally, hyphenated methods should provide the same quality of spectroscopic information as that acquired from isolated pure products. However, this is not the case, and each technique presents its limitations. The coupling of various hyphenated methods together (hypernation) requires even more compromises to be made (compatibility of the eluent composition, sensitivity and dynamic range of the methods being hypernated), but it has the advantage that all structural information is obtained within one single analysis [122].

For natural product analysis, the dereplication procedures rely mainly on HPLC-DAD-MS [82] and chemotaxonomic information provided by NP databases [123]. When unknown natural products cannot be dereplicated by these means, complementary information can be obtained by NMR either directly hyphenated with LC (HPLC-NMR) [124] or used at-line with preconcentration methods such as HPLC-SPE-NMR [125], [126] or after microfractionation with microflow HPLC-NMR methods such as CapNMR [119], or even with a combination of both techniques by CAP-SPE-NMR [127]. Other hyphenated methods, such as HPLC-IR [128] or HPLC-CD [129], can bring useful additional structural information.

The goal is not to cover HPLC hyphenation exhaustively here, as a dedicated review on plant fingerprinting can be found in this issue. However, the methods will be discussed briefly to complete the aspects regarding HPLC detection.

\section{HPLC-DAD (photodiode array detection)}

Photodiode array detection (DAD) [130] provides UV spectra directly online and is particularly useful for the detection of natural products with characteristic chromophores [131]. For example, polyphenols can be efficiently localised by this method because they possess characteristic chromophores. With this type of compound, DAD-UV spectral libraries can be built and used for dereplication, but compounds have to be analysed under the same HPLC conditions, as the composition of the mobile phase might affect the UV bands slightly. Furthermore, the choice of modifiers affects UV detection and might blind the bands in the low-wavelength range. The possibility to acquire several UV spectra across a given LC peak allows the assessment of peak purity [131]. HPLC-DAD analysis has been applied alone for the partial characterisation of many NPs with characteristic chromophores, mainly phenolics [132], polyketides, alkaloids, and terpenoids [131]. The technique also has been used successfully in combination with UV shift reagents, which are classically used for the structural characterisation of flavonoids in their pure form [119], [133]. Comparison of the genuine and shifted online DAD spectra enables a precise localisation of the hydroxy groups on the polyphenols online. Another interesting point regarding DAD-UV detection is that all wavelengths are stored during analysis, and thus multiple wavelengths can be monitored at the same time for detection of different classes of compounds. This has been found to be very useful for simultaneous detection of the naphthodianthrones $(590 \mathrm{~nm})$ and phloroglucinols $(270 \mathrm{~nm})$ of Hypericum perforatum [18] or for the relatively selective determination of glucosinolates $(227 \mathrm{~nm})$, indolic derivatives $(270 \mathrm{~nm})$, flavonoids and cinnamic derivatives $(325 \mathrm{~nm})$, and anthocyanidins (520 nm) in different Brassicaceae [134]. As for simple HPLC-UV, applications of HPLC-DAD in the analysis of NPs are manifold [82], [131] and cannot be extensively dis- 
cussed here. HPLC-DAD systems are commonly used in multihyphenated systems with MS [135] or ELSD [70] detection methods.

In Fig. 1 (inset a), an example of a UV-DAD spectrum of a flavonoid $(F)$ recorded online in the profiling of the constituents of Arabidopsis thaliana is shown. The presence of two maxima at 280 and $360 \mathrm{~nm}$ attributed, respectively, to the benzoic (band II) and cinnamoyl (band I) chromophore moieties was characteristic for this type of compound. The complete dereplication was achieved thanks to the complementary MS information obtained online (see below).

\section{HPLC-MS, MS-MS, and MSn}

HPLC-MS represents a key technique for the online identification of natural products. Besides detection, a mass spectrometer gives the possibility of generating either nominal mass molecular ions or accurate mass measurements for the determination of empirical formulas [86]. Furthermore, the use of tandem or hybrid MS instruments [80] provides in-depth structural information through fragmentation of the molecular species by CID reactions. Several reviews describe aspects related to online NP identification for screening and dereplication [82], [114], [115], [119], [136], for quality control, for fingerprinting for authentication or standardisation [80], or for the identification of biomarkers [137].

For online dereplication purposes, the determination of molecular weight is of great importance. This, however, necessitates the comparison of MS data obtained under different detection conditions in order to differentiate protonated or deprotonated molecules from adducts or fragments [119], [138]. The use of highresolution instruments, such as a TOF-MS [34], [103] or FT-MS [139], [140] system, enables direct determination of the molecular formula of crude mixtures. This strategic information allows for more precisely targeting the search in natural product libraries for dereplication purposes [119].

Complementary structural information can be generated by insource collision-induced dissociation (CID) in HPLC-MS-MS or MS $^{n}$ experiments [141]. The generated CID spectra are, however, not comparable to those recorded by EI, and this hampers a direct use of the standard EI-MS libraries for dereplication purposes. For performing automated dereplication procedures, specific MS-MS libraries have to be built based on standards available in a given laboratory, which consequently limits this approach. The development of hybrid instruments combined with high-resolution spectrometers, such as TOF, FT, or orbitrap analysers, allows the possibly to record MS-MS spectra with high mass accuracy, facilitating the interpretation of the obtained spectra [142].

In natural product chemistry, MS-MS spectra are mainly useful for the partial determination of sugar sequences of various glycosides [143]; the detection of characteristic losses, such as those of prenylated compounds [144]; the classic fragmentation of flavonoids or related compounds for the determination of substituent positions on A or B rings [145]; and the differentiation of isomers [120]. For other studies, the interpretation of the MS-MS spectra often requires the analysis of many related products [146] in order to extract structurally relevant information and establish rules that can be used for structure prediction. It is also worth mentioning that MS-MS is a well-established method for the analysis of peptides and provides unambiguous information on the sequence of amino acids based on precise fragmentation rules [147]. The method is also applicable to naturally occurring plant cyclic peptides after conversion into acy- clic forms by partial hydrolysis [148]. For the analysis of fully unknown constituents, MS-MS usually cannot provide enough information to ascertain structure determination, and thus the combination with other online information is mandatory.

In 0 Fig. 1 (inset b), an example of an MS spectrum obtained with ESI (PI) on a TOF instrument is presented for the accurate mass determination of a flavonoid triglycoside (F) (kaempferol 3-O- $\beta$-[ $\beta$-D-rhamnopyranosyl $(1 \rightarrow 2)$ D-glucopyranoside $]-7-O-\alpha$-Lrhamnopyranoside) of Arabidopsis thaliana. As shown, the $[\mathrm{M}+\mathrm{H}]^{+}$ion was recorded with high precision $(m / z=741.2242)$ with the help of a reference compound analysed during the whole procedure on a separated sprayer (LockSpray) [149]. This exact mass measurement enables the molecular formula determination $\left(\mathrm{C}_{33} \mathrm{H}_{41} \mathrm{O}_{19}\right)$ with an accuracy of less than $5 \mathrm{ppm}$. A cross-library search with this molecular formula and chemotaxonomic information (genus Arabidopsis) in a library of natural products (DNP Chapman and Hall [123]) enabled the dereplication of this compound. Its UV-DAD spectrum ( $\bullet$ Fig. 1, inset a) confirmed that this compound was a flavonol derivative (see above).

\section{HPLC-NMR (nuclear magnetic resonance)}

Because of the lack of efficient commercial MS-MS databases, the dereplication process often requires additional spectroscopic information to ascertain the identity of known natural products or to partially identify unknown metabolites. In this respect, HPLC-NMR provides a powerful tool that can yield important complementary information or, in some cases, a complete structural assignment of natural products online [119], [124], [150], [151]. HPLC-NMR should ideally enable the complete structural characterisation of any plant metabolite directly in an extract, provided that its corresponding LC peak is clearly resolved. In practice, however, this is not entirely true, as many factors hinder online structure determination [150]. These problems are linked mainly to the inherent low sensitivity of NMR for the detection of microgram or sub-microgram quantities of natural products separated by conventional HPLC and the need for solvent suppression.

Indeed, HPLC-NMR can be regarded as relatively insensitive ( $\odot$ Table 1) due to the intrinsic properties of NMR detection. Consequently, the LODs are several orders of magnitude higher than those of HPLC-UV or HPLC-MS. These limits also depend on the type of magnet used $(400-900 \mathrm{MHz})$ and the type of flow probe employed (standard HPLC-NMR probes $40-120 \mu \mathrm{L}$, microcoils $20 \mathrm{~nL}-1 \mu \mathrm{L}$ or cryo-flow probes). The mode of operation also strongly affects sensitivity. Indeed, in the on-flow measurements the time of acquisition is limited, and only few transients are recorded when the LC peak crosses the flow cell, while in the stop-flow mode an important number of transients can be acquired, consequently improving the $\mathrm{S} / \mathrm{N}$ ratio. With a standard HPLC-NMR flow probe ( $60 \mu \mathrm{L}$ on a $500-\mathrm{MHz}$ magnet), the detection limits are about $20 \mu \mathrm{g}$ of NP injected on-column in the on-flow mode, but they can reach the nanogram range when HPLC-NMR is used at-line with sensitive microflow probes [119].

HPLC-NMR, in the on-flow mode, can provide ${ }^{1} \mathrm{H}-\mathrm{NMR}$ spectra of the main constituent of a crude extract rapidly without the need for complex automation. This type of information, in addition to other online spectroscopic data (e.g., DAD and MS), may ascertain the structural determination and facilitate the dereplication process. Examples of on-flow HPLC-NMR analyses for crude extract profiling are manifold (e.g., for flavonoids, alkaloids, ter- 
penes, and carotenoids) [119], [121], [124], although a relatively limited number of NPs, essentially only the very major ones, are detected in this way. The technique is also extremely useful for detecting compounds that are labile or might epimerise or interconvert as a result of their isolation [152].

One of the limiting factors of online HPLC-NMR is the need for solvent suppression, because HPLC separation is performed with $\mathrm{MeCN}-\mathrm{D}_{2} \mathrm{O}$ and $\mathrm{MeOH}-\mathrm{D}_{2} \mathrm{O}$. In practice, the very large signal of $\mathrm{MeCN}$ or $\mathrm{MeOH}$ and the residual HOD signal are completely eliminated by powerful solvent suppression sequences. The main drawback of this procedure is that analyte signals localised under the solvent resonances also will be suppressed, and thus some information can potentially be lost. Another problem occurring in HPLC-NMR is that the chemical shifts recorded in a typical reversed-phase solvent will differ from those reported in standard deuterated NMR solvents. This can be a drawback if precise comparisons with literature data have to be performed [150].

In order to circumvent these problems, new approaches in this field allow use of HPLC-NMR at-line instead of online. In this case, trapping the HPLC peaks on SPE for preconcentration prior to NMR detection can be performed (SPE-NMR) [125], [153]. Another alternative is HPLC microfractionation of the extract, drying, and re-injection of the concentrated HPLC peak in deuterated solvent and using microflow capillary HPLC-NMR probes (CapNMR) [154], [155], [156]. These at-line HPLC-NMR analyses provide a good HPLC peak preconcentration and high quality $1 \mathrm{D}$ and $2 \mathrm{D}$ NMR spectra in fully deuterated solvents without the need for solvent suppression. The work at-line has the disadvantage that more sample handling or automation is needed compared with the on-flow approach. However, these techniques are crucial for the complete de novo structure elucidation of given HPLC peaks, as they can provide ${ }^{1} \mathrm{H}^{13} \mathrm{C}$ correlation experiments in the low microgram range [150].

\section{Detection without HPLC separation}

With the important development of multivariate data analysis techniques, the spectroscopic methods used for HPLC detection (mainly NMR, IR, and MS) are more often being applied to the analysis of crude extracts without prior HPLC separation for a rapid evaluation of the composition, especially in the quality control of herbal medical products and metabolomics [157]. For example, based on the quality control examples discussed previously for Gingko, ginseng, and Hypericum, alternative methods not requiring HPLC separation have been reported.

In the case of Ginkgo biloba, the determination of the content in allergenic ginkgolic acids has been evaluated by direct ${ }^{1} \mathrm{H}-\mathrm{NMR}$ analysis of various commercial preparations without any prepurification steps. The aromatic protons common to all of these compounds were well separated from other signals in the total ${ }^{1} \mathrm{H}$-NMR spectra of the extracts. The quantity of the compounds was calculated from the relative ratio of the integral of these NMR peaks with an internal standard, and the results were comparable with other chromatographic methods of quantification [158]. Similarly for Hypericum perforatum, hypericin and its derivative pseudohypericin were efficiently quantified by ${ }^{1} \mathrm{H}-\mathrm{NMR}$ with the specific detection of strongly de-shielded peri-hydroxyl protons of hypericins in the region of $14-15 \mathrm{ppm}$ in $\mathrm{CD}_{3} \mathrm{OH}$ solutions [159]. The high-throughput analysis of 80 phytopreparations of the same plant was also efficiently performed with a near-infrared spectroscopic method. Hypericin, pseudohypericin, and the phloroglucine derivative hyperforin were quantified simultaneously after subsequent chemometric calculations in very short analysis times of a few seconds [160]. In the case of ginseng, metabolic fingerprinting of different preparations was performed by ${ }^{1} \mathrm{H}-\mathrm{NMR}$ spectroscopy in less than 15 minutes without any pre-purification steps. Improvement in the resolution of the ${ }^{1} \mathrm{H}-\mathrm{NMR}$ spectra was obtained by two-dimensional Jresolved NMR measurements. MVDA was applied for the analysis, and PCA showed a clear discrimination among the samples. The results demonstrate that not only ginsenosides but also alanine, arginine, choline, fumaric acid, inositol, and sucrose differentiate the preparations from each other [161]. For other medicinal plants, such as Galphimia glauca, popularly employed in Mexico, MVDA applied to crude extracts from different wild plant populations provided a good mean to assess quality and enabled a linkage of the neuropharmacological effects to given chemical profiles [162].

Thus, as shown, direct detection of given metabolites also can be performed without HPLC separation. Such approaches are not applicable to any type of samples and require that specific signals can be localised within the total extract spectra. However, they represent very interesting complements to the classical HPLC profiling approaches.

\section{Conclusions}

$\nabla$

The quest for universal and sensitive HPLC detection with uniform response factors for all analytes is ongoing. Thus far, no ideal solution has been found, and specific detectors need to be chosen according to the type of NP analysed. The lack of a sensitive and universal detection technique suitable for both qualitative and quantitative analysis under a wide range of conditions is one of the reasons why the development of generally applicable methods for HPLC is still challenging. As shown, many LC detectors have been developed over the years, with different advantages and limitations, and a summary of their main features is provided in Table 2. A literature search restricted to journals focused on NP studies revealed that more than 70\% of HPLC applications are performed by HPLC-UV and HPLC-DAD, with HPLC-MS applications accounting for about 20\% (one-third being related to MS-MS), HPLC-ECD for $3 \%$, HPLC-NMR for $2 \%$, and ELSD for $1.5 \%$. The other detection techniques are reported in less than $1 \%$ of all HPLC applications. The large majority of analyses are still performed by HPLC-UV, which is widespread in many laboratories. For the detection of non-UV-active constituents, aerosol-based detectors, such as ELSD or CAD, represent a cost-effective alternative to HPLC-MS and will probably increase in popularity. HPLC-MS is a very versatile and powerful tool. It is sensitive and can be considered both a specific and a universal detection method. The technique is still expensive, but the development of benchtop instruments that are easy to operate will continue to increase its use in many areas of NP research. However, quantitative analysis with HPLC-MS is still less robust than with other techniques, as the response depends on the ionisation process and the method suffers from ionisation suppression problems when complex matrices are analysed.

The introduction of sub $2-\mu \mathrm{m}$ columns for HPLC and the development of UHPLC represent important steps forward for crude extract profiling. The speed of separation provided by these new separation methods challenges the LC detectors, which have to provide faster responses. The strong development of 
Table 2 Summary of the advantages and limitations of each LC detection method

\begin{tabular}{|c|c|c|c|}
\hline & Principal advantages & Principal limitations & Application to NPs \\
\hline UV & $\begin{array}{l}\text { Widespread, cheap, easy to use, adapted } \\
\text { to a relatively large number of NPs with } \\
\text { detection at low wavelengths }\end{array}$ & $\begin{array}{l}\text { Not applicable to compounds without } \\
\text { chromophores, not very selective, not } \\
\text { compatible with mobile-phase } \\
\text { constituents exhibiting high UV cut-offs }\end{array}$ & $\begin{array}{l}\text { All NPs with chromophores (even weak } \\
\text { chromophores: } 203 \mathrm{~nm} \text { ) }\end{array}$ \\
\hline DAD & $\begin{array}{l}\text { Recording of all wavelengths, possibility } \\
\text { to edit given spectra for dereplication, } \\
\text { monitoring of different wavelengths at } \\
\text { the same time }\end{array}$ & $\begin{array}{l}\text { Structural information by UV spectra is } \\
\text { limited for dereplication purposes }\end{array}$ & $\begin{array}{l}\text { All NPs with characteristic chromophores, } \\
\text { especially polyphenols, alkaloids, } \\
\text { quinones, and polyketides }\end{array}$ \\
\hline FD & High sensitivity and selectivity & $\begin{array}{l}\text { Limited to NPs with natural fluorescence } \\
\text { or need for tedious derivatisation } \\
\text { procedure }\end{array}$ & $\begin{array}{l}\text { Mainly aflatoxins, selective detection } \\
\text { of other NPs after derivation with } \\
\text { fluorescent tags }\end{array}$ \\
\hline EC & Cheap, sensitive, and relatively selective & $\begin{array}{l}\text { Need optimisation of the potential used } \\
\text { and knowledge of redox reactions } \\
\text { involved, detection alters the sample }\end{array}$ & $\begin{array}{l}\text { All NPs having electroactive groups, such } \\
\text { as phenols, aromatic, amines, thiols, } \\
\text { olefins, ester, ketones, aldehydes, ethers, } \\
\text { and quinones }\end{array}$ \\
\hline RID & Universal, cheap & $\begin{array}{l}\text { Low sensitivity, not applicable to } \\
\text { gradient, sensitive to changes in } \\
\text { temperature, pressure, and flow rate }\end{array}$ & $\begin{array}{l}\text { Carbohydrates, polymers, and some } \\
\text { terpenes }\end{array}$ \\
\hline ELSD & $\begin{array}{l}\text { Universal, widespread, easy to use, } \\
\text { compatible with gradient elution }\end{array}$ & $\begin{array}{l}\text { Non-linear response, need optimisation } \\
\text { of gas flow and drift tube temperature }\end{array}$ & $\begin{array}{l}\text { All NPs, mainly used for the detection of } \\
\text { NPs with weak chromophores such as } \\
\text { terpenes and carbohydrates }\end{array}$ \\
\hline CAD & $\begin{array}{l}\text { Universal, more sensitive than ELSD, } \\
\text { large dynamic range ( } 4 \text { orders of } \\
\text { magnitude) }\end{array}$ & Non-linear response & $\begin{array}{l}\text { Not yet applied to NPs, used for pharma- } \\
\text { ceuticals, polysaccharides, and lipids }\end{array}$ \\
\hline MS & $\begin{array}{l}\text { Universal, widespread, specific, } \\
\text { sensitive, provides structural MW } \\
\text { information (LR mode) or molecular } \\
\text { formula (HR mode) }\end{array}$ & $\begin{array}{l}\text { Compound-dependent response, not } \\
\text { compatible with non-volatile buffers, } \\
\text { sensitive to ion suppression effects in } \\
\text { complex matrices, expensive }\end{array}$ & $\begin{array}{l}\text { All NPs, mainly large MW and polar: } \\
\text { ESI, mainly medium MW and medium } \\
\text { polarity: APCI, low sensitivity for non- } \\
\text { polar NPs }\end{array}$ \\
\hline MS-MS & $\begin{array}{l}\text { Universal, widespread, sensitive, } \\
\text { provides fragment information and very } \\
\text { specific detection (MRM mode) }\end{array}$ & $\begin{array}{l}\text { Same as MS, instrument dependence of } \\
\text { the MS-MS spectra, lack of MS-MS } \\
\text { database for NPs }\end{array}$ & $\begin{array}{l}\text { Same as MS, fragment information brings } \\
\text { useful information mainly for glycosides } \\
\text { and/or polyphenols }\end{array}$ \\
\hline NMR & $\begin{array}{l}\text { Universal, important structural } \\
\text { information for de novo structure } \\
\text { determination online }\end{array}$ & $\begin{array}{l}\text { Low sensitivity, response depends on } \\
\text { the operation mode (on-flow, stop-flow, } \\
\text { at-line), expensive }\end{array}$ & $\begin{array}{l}\text { All NPs, on-flow for the detection of NPs } \\
\text { with characteristic }{ }^{1} \mathrm{H} \text { pattern, at-line for } \\
\text { in-depth structure determination ( } 1 \mathrm{D} \\
\text { and 2 D) }\end{array}$ \\
\hline
\end{tabular}

“-omic's” approaches in NP research (metabolomics, genomics, etc.) over the last decade also challenges the analytical methods as they aim to detect and quantify all metabolites in a given organism. For these types of studies, both separative and nonseparative methods must be complementary because none of them alone can fulfil all the ideal needs of sensitivity and throughput.

For online structural determination, HPLC-DAD-MS represents a key strategic tool, and it is used mainly for dereplication purposes in laboratories involved in drug discovery programs. However, the lack of general HPLC-MS databases restricts the possibility of prediction and automation in the dereplication process. Thus, complementary methods such as HPLC-NMR and derived techniques used at-line (SPE-NMR or CapNMR) are necessary. A topic not discussed in this review is the use of biological assays for HPLC detection in drug discovery. Many developments have been made in this area during recent years, especially for HPLCbased activity profiling and HPLC-based on-flow bioassays [163]. These types of approaches are becoming more integrated with multi-hyphenated methods, providing completely hyphenated and integrated lead-finding platforms.

With the increasing requirements for quality control, profiling and fingerprinting, dereplication, and metabolomics, the detection issue in HPLC is still challenging. New detectors still have to be developed to fulfil the needs of sensitivity, linearity, speed, robustness, and universal response requested by the investigations performed currently in natural products research.

\section{Acknowledgements}

The author is grateful to Davy Guillarme, Gaetan Glauser, and Elia Grata for their critical comments on the manuscript and for providing the data used to generate 0 Fig. 1. For the profiling of Arabidopsis constituents, financial support was provided by the Swiss National Science Foundation (grant no. 205320-107 735/1 to J-L.W.)

\section{References}

1 Larsson J, Gottfries J, Muresan S, Backlund A. ChemGPS-NP: Tuned for navigation in biologically relevant chemical space. J Nat Prod 2007; 70: $789-94$

2 Rochfort S. Metabolomics reviewed: A new "Omics" platform technology for systems biology and implications for natural products research. J Nat Prod 2005; 68: $1813-20$

3 Kingston DGI. High-performance liquid-chromatography of naturalproducts. J Nat Prod 1979; 42: 237-60

4 Natishan TK. Recent developments of achiral HPLC methods in pharmaceuticals using various detection modes. J Liq Chromatogr Relat Technol 2004; 27: 1237-316

5 Nguyen DTT, Guillarme D, Rudaz S, Veuthey JL. Fast analysis in liquid chromatography using small particle size and high pressure. J Sep Sci 2006; 29: $1836-48$ 
6 David F, Vanhoenacker G, Tienpont B, Francois I, Sandra P. Coupling columns and multidimensional configurations to increase peak capacity in liquid chromatography. Lc Gc Europe 2007; 20: $154-8$

7 Wilson ID, Brinkman UAT. Hyphenation and hypernation - The practice and prospects of multiple hyphenation. J Chromatogr A 2003; 1000: $325-56$

8 Scott RPW. Liquid chromatography detectors. Available at Chrom-Ed Book Series: Library4Science, http://www.library4science.com/; Accessed 2003

9 Gao XF, Dan M, Zhao AH, Xie GX, Jia W. Simultaneous determination of saponins in flower buds of Panax notoginseng using high performance liquid chromatography. Biomed Chromatogr 2008; 22: 244-9

10 Ozkan SA. LC with electrochemical detection. recent application to pharmaceuticals and biological fluids. Chromatographia 2007; 66: S3-S13

11 Vial J, Jardy A. Study of the linear range in HPLC analyses with UV detection: Methodology and experimental application to the influence of the analyte UV spectrum. J High Resol Chromatogr 1999; 22: 217-21

12 Leitner A, Emmert J, Boerner K, Lindner W. Influence of solvent additive composition on chromatographic separation and sodium adduct formation of peptides in HPLC-ESI MS. Chromatographia 2007; 65: 649-53

13 Cimpan G, Gocan S. Analysis of medicinal plants by HPLC: Recent approaches. J Liq Chromatogr Relat Technol 2002; 25: 2225 - 92

14 United States Pharmacopeial Convention. The United States pharmacopeia USP 31 NF 26. Rockville, MD: United States Pharmacopeial Convention; 2007: 3 volumes

15 European Pharmacopoeia 5.1, 5th edition. Strasbourg: Council of Europe; 2002

16 Fuzzati N. Analysis methods of ginsenosides. J Chromatogr B 2004; 812: $119-33$

17 Brolis M, Gabetta B, Fuzzati N, Pace R, Panzeri F, Peterlongo F. Identification by high-performace liquid chromatography-diode array detection-mass spectrometry and quantification by high performance liquid chromatography-UV absorbance detection of active constituents of Hypericum perforatum. J Chromatogr A 1998; 825: 9-16

18 Tolonen A, Hohtola A, Jalonen J. Fast high-performance liquid chromatographic analysis of naphthodianthrones and phloroglucinols from Hypericum perforatum extracts. Phytochem Anal 2003; 14: 306-9

19 Hasler A, Sticher O, Meier B. Identification and determination of the flavonoid from Ginkgo biloba by high performance liquid chromatography. J Chromatogr 1992; 605: $41-8$

20 Dubber MJ, Kanfer I. High-performance liquid chromatographic determination of selected flavonols in Ginkgo biloba solid oral dosage forms. J Pharm Pharm Sci 2004; 7: 303-9

21 Sloley BD, Tawfik SR, Scherban KA, Tam YK. Quality control analyses for ginkgo extracts require analysis of intact flavonol glycosides. J Food Drug Anal 2003; 11: $102-7$

22 Chen P, Ozcan M, Harnly J. Chromatographic fingerprint analysis for evaluation of Ginkgo biloba products. Anal Bioanal Chem 2007; 389: $251-61$

23 Jensen AG, Ndjoko K, Wolfender JL, Hostettmann K, Camponovo F, Soldati F. Liquid chromatography-atmospheric pressure chemical ionisation/mass spectrometry: A rapid and selective method for the quantitative determination of ginkgolides and bilobalide in Ginkgo leaf extracts and phytopharmaceuticals. Phytochem Anal 2002; 13 : $31-8$

24 Li WK, Fitzloff JF. HPLC determination of flavonoids and terpene lactones in commercial Ginkgo biloba products. J Liq Chromatogr Relat Technol 2002; 25: 2501 - 14

25 Schotz K. Quantification of allergenic urushiols in extracts of Ginkgo biloba leaves, in simple one-step extracts and refined manufactured material (EGb 761). Phytochem Anal 2004; 15: 1 - 8

26 Ndjoko K, Wolfender JL, Hostettmann $K$. Determination of trace amounts of ginkgolic acids in Ginkgo biloba L. leaf extracts and phytopharmaceuticals by liquid chromatography-electrospray mass spectrometry. J Chromatogr B 2000; 744: 249-55

27 Fuzzati N, Pace R, Villa E. A simple HPLC-UV method for the assay of ginkgolic acids in Ginkgo biloba extracts. Fitoterapia 2003; 74: 247-56

28 Mohn T, Potterat $O$, Hamburger $M$. Quantification of active principles and pigments in leaf extracts of Isatis tinctoria by HPLC/UV/MS. Planta Med 2007; 73: 151 - 6

29 Bebrevska L, Bravo L, Vandervoort J, Pieters L, Vlietinck A, Apers S. Development and validation of an HPLC method for quality control of Pueraria lobata flower. Planta Med 2007; 73: 1606-13
30 Avula B, Joshi VC, Reddy NVL, Choi YW, Khan LA. Simultaneous determination of eight coumarins in Angelica gigas and in various other Angelica species by high performance liquid chromatography and comparative micro-morphology study of Angelica species. Planta Med 2007; 73: 1509-16

31 Liang Z, Jiang Z, Ho H, Zhao Z. Comparative analysis of Oldenlandia diffusa and its substitutes by high performance liquid chromatographic fingerprint and mass spectrometric analysis. Planta Med 2007; 73: $1502-8$

32 Ge GB, Zhang YY, Hao DC, Hu Y, Luan HW, Liu XB et al. Chemotaxonomic study of medicinal Taxus species with fingerprint and multivariate analysis. Planta Med 2008; 74: 773 -9

33 Grata E, Boccard J, Glauser G, Carrupt PA, Farmer EE, Wolfender JL et al. Development of a two-step screening ESI-TOF-MS method for rapid determination of significant stress-induced metabolome modifications in plant leaf extracts: The wound response in Arabidopsis thaliana as a case study. J Sep Sci 2007; 30: 2268 - 78

34 Grata E, Boccard J, Guillarme D, Glauser G, Carrupt PA, Farmer E et al. UPLC-TOF-MS for plant metabolomics: A sequential approach for wound marker analysis in Arabidopsis thaliana. J Chromatogr B 2008; 871: $261-70$

35 Jaimez J, Fente CA, Vazquez BI, Franco CM, Cepeda A, Mahuzier G et al. Application of the assay of aflatoxins by liquid chromatography with fluorescence detection in food analysis. J Chromatogr A 2000; 882: 1 10

36 Braga S, de Medeiros FD, Oliveira ED, Macedo RO. Development and validation of a method for the quantitative determination of aflatoxin contaminants in Maytenus ilicifolia by HPLC with fluorescence detection. Phytochem Anal 2005; 16: 267 - 71

37 Klvana M, Chen JK, Lepine F, Legros R, Jolicoeur M. Analysis of secondary metabolites from Eschscholtzia californica by high-performance liquid chromatography. Phytochem Anal 2006; 17: 236 - 42

38 Kristl J, Veber M, Krajnicic B, Oresnik K, Slekovec M. Determination of jasmonic acid in Lemna minor (L.) by liquid chromatography with fluorescence detection. Anal Bioanal Chem 2005; 383: 886-93

39 Li FM, Zhang CH, Guo XJ, Feng WY. Chemiluminescence detection in HPLC and CE for pharmaceutical and biomedical analysis. Biomed Chromatogr 2003; 17: 96-105

40 Ohba Y, Kuroda N, Nakashima K. Liquid chromatography of fatty acids with chemiluminescence detection. Anal Chim Acta 2002; 465: 101 -

41 Zhang QL, Cui H. Simultaneous determination of quercetin, kaempferol, and isorhamnetin in phytopharmaceuticals of Hippophae rhamnoides L. by high-performance liquid chromatography with chemiluminescence detection. J Sep Sci 2005; 28: 1171-8

42 LaCourse WR, Modi SJ. Microelectrode applications of pulsed electrochemical detection. Electroanalysis 2005; 17: 1141 - 52

43 Guo CJ, Cao GH, Sofic E, Prior RL. High-performance liquid chromatography coupled with coulometric array detection of electroactive components in fruits and vegetables: Relationship to oxygen radical absorbance capacity. J Agric Food Chem 1997; 45: 1787-96

44 Wang CY, Xu JY, Zhou GY, Qu QS, Yang GJ, Hu XY. Electrochemical detection coupled with high-performance liquid chromatography in pharmaceutical and biomedical analysis: A mini review. Comb Chem High Throughput Screen 2007; 10: 547 - 54

45 Skrinjar M, Kolar MH, Jelsek N, Hras AR, Bezjak M, Knez Z. Application of HPLC with electrochemical detection for the determination of low levels of antioxidants. J Food Comp Anal 2007; 20: 539 - 45

46 Ma LJ, Zhang XZ, Zhang HP, Gan YR. Development of a fingerprint of Salvia miltiorrhiza Bunge by high-performance liquid chromatography with a coulometric electrode array system. J Chromatogr B 2007; 846: $139-46$

47 Casella IG, Colonna C, Contursi M. Electroanalytical determination of some phenolic acids by high-performance liquid chromatography at gold electrodes. Electroanalysis 2007; 19: 1503-8

48 Zhou Q Zhu YX, Hwa CA, Yagiz K, Morre DJ, Morre DA et al. Identification of the major vandloid component in Capsicum extract by HPLCEC and HPLC-MS. Phytochem Anal 2004; 15: 117-20

49 Liu ZH, Sang SM, Hartman TG, Ho CT, Rosen RT. Determination of diarylheptanoids from Alpinia officinarum (lesser galangal) by HPLC with photodiode array and electrochemical detection. Phytochem Anal 2005; 16: $252-6$

50 Ruckert $U$, Likussar W, Michelitsch A. Simultaneous determination of total hypericin and hyperforin in St. John's wort extracts by HPLC with electrochemical detection. Phytochem Anal 2007; 18: 204-8 
51 Aaby K, Hvattum E, Skrede G. Analysis of flavonoids and other phenolic compounds using high-performance liquid chromatography with coulometric array detection: Relationship to antioxidant activity. J Agric Food Chem 2004; 52: 4595-603

52 Chan KL, Yuen KH, Jinadasa S, Peh KK, Toh WT. A high-performance liquid chromatography analysis of plasma artemisinin using a glassy carbon electrode for reductive electrochemical detection. Planta Med 1997; 63: $66-9$

53 Wu YT, Tsai TR, Lin LC, Tsai TH. Liquid chromatographic method with amperometric detection to determine acteoside in rat blood and brain microdialysates and its application to pharmacokinetic study. J Chromatogr B 2007; 853: $281-6$

54 Joo KM, Park CW, Jeong HJ, Lee SJ, Chang IS. Simultaneous determination of two Amadori compounds in Korean red ginseng (Panax ginseng) extracts and rat plasma by high-performance anion-exchange chromatography with pulsed amperometric detection. J Chromatogr B 2008; 865: 159-66

55 Tiselius A, Claesson S. Adsorption analysis by means of interferometric study. Arkiv Kemi Minearl Geol 1942; 15: 1 - 6

56 Perez AG, Olias R, Espada J, Olias JM, Sanz C. Rapid determination of sugars, nonvolatile acids, and ascorbic acid in strawberry and other fruits. J Agric Food Chem 1997; 45: 3545 - 9

57 von Eggelkraut-Gottanka SG, Abu Abed S, Muller W, Schmidt PC. Quantitative analysis of the active components and the by-products of eight dry extracts of Hypericum perforatum L. (St John's wort). Phytochem Anal 2002; 13: 170-6

58 Ding C, Chen EQ Zhou WJ, Lindsay RC. A method for extraction and quantification of Ginkgo terpene trilactones. Anal Chem 2004; 76: 4332-6

59 Ford DL, Kennard W. Vaporization analyzer. J Oil Colour Chem Assoc 1966; 49: $299-313$

60 Megoulas NC, Koupparis MA. Twenty years of evaporative light scattering detection. Crit Rev Anal Chem 2005; 35: 301-16

61 Guillarme D, Rudaz S, Schelling C, Dreux M, Veuthey JL. Micro liquid chromatography coupled with evaporative light scattering detector at ambient and high temperature: Optimization of the nebulization cell geometry. J Chromatogr A 2008; 1192: $103-12$

62 Dubber MJ, Kanfer I. Determination of terpene trilactones in Ginkgo biloba solid oral dosage forms using HPLC with evaporative light scattering detection. J Pharm Biomed Anal 2006; 41: 135-40

63 Vervoort N, Daemen D, Torok G. Performance evaluation of evaporative light scattering detection and charged aerosol detection in reversed phase liquid chromatography. J Chromatogr A 2008; 1189: 92 - 100

64 Gorecki T, Lynen F, Szucs $R$, Sandra P. Universal response in liquid chromatography using charged aerosol detection. Anal Chem 2006; 78: $3186-92$

65 Russo R, Guillarme D, Bicchi C, Rudaz S, Veuthey JL. Evaluation of the coupling between ultra performance liquid chromatography and evaporative light scattering detector for selected phytochemical applications. J Sep Sci 2008; 31: 2377-87

66 Li P, Zeng LJ, Li SL, Lin G. The extraction of imperialine and imperialine3 beta-glucoside from Fritillaria pallidiflora Schrenk and quantitative determination by HPLC-evaporative light scattering detection. Phytochem Anal 2002; 13: 158 -61

67 Kim SN, Ha YW, Shin H, Son SH, Wu SJ, Kim YS. Simultaneous quantification of 14 ginsenosides in Panax ginseng C.A. Meyer (Korean red ginseng) by HPLC-ELSD and its application to quality control. J Pharm Biomed Anal 2007; 45: $164-70$

68 Schaneberg BT, Molyneux RJ, Khan IA. Evaporative light scattering detection of pyrrolizidine alkaloids. Phytochem Anal 2004; 15: 36-9

69 Cremin PA, Zeng L. High-throughput analysis of natural product compound libraries by parallel LC-MS evaporative light scattering detection. Anal Chem 2002; 74: $5492-500$

70 Li J, Qi H, Qi LW, Yi L, Li P. Simultaneous determination of main phytoecdysones and triterpenoids in Radix Achyranthis Bidentatae by high-performance liquid chromatography with diode array-evaporative light scattering detectors and mass spectrometry. Anal Chim Acta 2007; 596: $264-72$

71 Camponovo FF, Wolfender JL, Maillard MP, Potterat O, Hostettmann K. Evaporative light-scattering and thermospray mass-spectrometry Alternative methods for detection and quantitative liquid-chromatographic determination of ginkgolides and bilobalide in Ginkgo biloba leaf extracts and phytopharmaceuticals. Phytochem Anal 1995; 6: $141-8$

72 Deng FX, Zito SW. Development and validation of a gas chromatographic-mass spectrometric method for simultaneous identification and quantification of marker compounds including bilobalide, ginkgolides and flavonoids in Ginkgo biloba L. extract and pharmaceutical preparations. J Chromatogr A 2003; 986: 121 - 7

73 Dixon RW, Peterson DS. Development and testing of a detection method for liquid chromatography based on aerosol charging. Anal Chem 2002; 74: $2930-7$

74 Gamache PH, McCarthy RS, Freeto SM, Asa DJ, Woodcock MJ, Laws K et al. HPLC analysis of non-volatile analytes using charged aerosol detection. Lc Gc Europe 2005; 18: 345 -9

75 Lisa M, Lynen F, Holcapek M, Sandra P. Quantitation of triacylglycerols from plant oils using charged aerosol detection with gradient compensation. J Chromatogr A 2007; 1176: 135-42

76 Guillarme D, Heinisch S, Gauvrit JY, Lanteri P, Rocca JL. Optimization of the coupling of high-temperature liquid chromatography and flame ionization detection application to the separations of alcohols. J Chromatogr A 2005; 1078: 22 - 7

77 Foster MD, Synovec RE. Reversed phase liquid chromatography of organic hydrocarbons with water as the mobile phase. Anal Chem 1996; 68 : $2838-44$

78 Smith RM. Superheated water chromatography - A green technology for the future. J Chromatogr A 2008; 1184: 441 - 55

79 Guillarme D, Heinisch S. Detection modes with high temperature liquid chromatography - A review. Sep Purif Rev 2005; 34: 181 - 216

80 Cheng KW, Cheng F, Wang M. Liquid chromatography-mass spectrometry in natural product research. In: Colegate SM, Molyneux RJ, editors. Bioactive natural products: detection, isolation, and structural determination, 2nd edition. London: CRC press; 2008: 245 66

81 Niessen WMA. Liquid chromatography - mass spectrometry, 3 rd edition. Boca Raton: Taylor \& Francis; 2006: 608 S

$82 \mathrm{He}$ XG. On-line identification of phytochemical constituents in botanical extracts by combined high-performance liquid chromatographicdiode array detection-mass spectrometric techniques. J Chromatogr A 2000; 880: $203-32$

83 Games DE, Martinez F. Evaluation of the moving belt as an interface for high performance liquid chromatographic mass-spectrometric analysis of flavonoids aglycones. J Chromatogr 1989; 474: 372-80

84 Brewer TM, Castro J, Marcus RK. Particle beam sample introduction into glow discharge plasmas for speciation analysis. Spectrochim Acta Part B At Spectrosc 2006; 61: 134-49

85 Cappiello A, Famiglini G, Pierini E, Palma P, Trufelli H. Advanced liquid chromatography-mass spectrometry interface based on electron ionization. Anal Chem 2007; 79: 5364-72

86 Korfmacher WA. Principles and applications of LC-MS in new drug discovery. Drug Discov Today 2005; 10: 1357-67

87 Pisitkun T, Hoffert JD, Yu MJ, Knepper MA. Tandem mass spectrometry in physiology. Physiology 2007; 22: $390-400$

88 Williamson LN, Bartlett MG. Quantitative liquid chromatography/ time-of-flight mass spectrometry. Biomed Chromatogr 2007; 21: $567-76$

89 Jessome LL, Volmer DA. Ion suppression: A major concern in mass spectrometry. Lc Gc North America; 2006: 83 -9

90 Syage JA, Short LC, Cai SS. Atmospheric pressure photoionization - The second source for LC-MS? Lc Gc North America 2008; 26: 286 - 300

91 Jean-Denis JB, Pezet $R$, Tabacchi R. Rapid analysis of stilbenes and derivatives from downy mildew-infected grapevine leaves by liquid chromatography-atmospheric pressure photoionisation mass spectrometry. J Chromatogr A 2006; 1112: $263-8$

92 Gomez-Ariza JL, Garcia-Barrera T, Lorenzo F. Anthocyanins profile as fingerprint of wines using atmospheric pressure photoionisation coupled to quadrupole time-of-flight mass spectrometry. Anal Chim Acta 2006; 570: $101-8$

93 Cai YX, Kingery D, McConnell O, Bach AC. Advantages of atmospheric pressure photoionization mass spectrometry in support of drug discovery. Rapid Commun Mass Spectrom 2005; 19: 1717-24

94 Li L, Zhang JL, Sheng YX, Guo DA, Wang Q, Guo HZ. Simultaneous quantification of six major active saponins of Panax notoginseng by highperformance liquid chromatography-UV method. J Pharm Biomed Anal 2005; 38: $45-51$

95 Wang CZ, Aung HH, Ni M, Wu JA, Tong RB, Wicks S et al. Red American ginseng: Ginsenoside constituents and antiproliferative activities of heat-processed Panax quinquefolius roots. Planta Med 2007; 73: 669-74

96 Wan JB, Yang FQ Li SP, Wang YT, Cui XM. Chemical characteristics for different parts of Panax notoginseng using pressurized liquid extraction and HPLC-ELSD. J Pharm Biomed Anal 2006; 41: 1596-601 
97 Fuzzati N, Gabetta B, Jayakar K, Pace R, Peterlongo F. Liquid chromatography-electrospray mass spectrometric identification of ginsenosides in Panax ginseng roots. J Chromatogr A 1999; 854: 69-79

98 Luchtefeld R, Kostoryz E, Smith RE. Determination of ginsenosides Rb-1, $\mathrm{R}-\mathrm{C}$, and $\mathrm{R}$-e in different dosage forms of ginseng by negative ion electrospray liquid chromatography-mass spectrometry. J Agric Food Chem 2004; 52: 4953-6

99 Kite GC, Howes MJR, Leon CJ, Simmonds MSJ. Liquid chromatography/ mass spectrometry of malonyl-ginsenosides in the authentication of ginseng. Rapid Commun Mass Spectrom 2003; 17: 238 - 44

100 Wang X, Sakuma T, Asajufa-adjaye E, Shiu G. Determination of ginsenosides in plant extracts from Panax ginseng and Panax quinquefolius by LC/MS/MS. Anal Chem 1999; 71: 1579-84

101 Wang Y, Pan JY, Xiao XY, Lin RC, Cheng YY. Simultaneous determination of ginsenosides in Panax ginseng with different growth ages using high-performance liquid chromatography-mass spectrometry. Phytochem Anal 2006; 17: 424-30

102 Ligor T, Ludwiczuk A, Wolski T, Buszewski B. Isolation and determination of ginsenosides in American ginseng leaves and root extracts by LC-MS. Anal Bioanal Chem 2005; 383: 1098 - 105

103 Xie GX, Plumb R, Su MM, Xu ZH, Zhao AH, Qiu MF et al. Ultra-performance LC/TOF MS analysis of medicinal Panax herbs for metabolomic research. J Sep Sci 2008; 31: 1015 - 26

104 Ma X, Xiao HB, Liang XM. Identification of ginsenosides in Panax quinquefolium by LC-MS. Chromatographia 2006; 64: 31 -6

105 Yu ZG, Gao XX, Zhao YL, Chen XH, Bi KS. Simultaneous determination of components in preparation Naodesheng injection by high performance liquid chromatograph-atmospheric pressure chemical ionization mass spectrometry (HPLC-MS/APCI). Chem Pharm Bull 2006; 54: $588-90$

106 Wang W, Wang GJ, Xie HT, Sun JG, Zhao S, Jiang XL et al. Determination of ginsenoside Rd in dog plasma by liquid chromatography-mass spectrometry after solid-phase extraction and its application in dog pharmacokinetics studies. J Chromatogr B 2007; 852: 8-14

107 Liu H, Huang Y, Wang $Q$ Z Zhang T, Song Y. Detection of saponins in extracts from the rhizomes of Paris species and prepared Chinese medicines by high performance liquid chromatography-electrospray ionization mass spectrometry. Planta Med 2006; 72: 835-41

108 Sun YK, Li WK, Fitzloff JF, van Breemen RB. Liquid chromatography/ electrospray tandem mass spectrometry of terpenoid lactones in Ginkgo biloba. J Mass Spectrom 2005; 40: 373 -9

109 Xie JS, Ding CG, Ge QH, Zhou Z, Zhi XJ. Simultaneous determination of ginkgolides A, B, C and bilobalide in plasma by LC-MS/MS and its application to the pharmacokinetic study of Ginkgo biloba extract in rats. J Chromatogr B 2008; 864: 87-94

110 Draper WM, Wijekoon D, McKinney M, Behniwal P, Perera SK, Flessel $C P$. Atmospheric pressure ionization LC-MS-MS determination of urushiol congeners. J Agric Food Chem 2002; 50: 1852 -8

111 Betteridge K, Cao Y, Colegate SM. Improved method for extraction and LC-MS analysis of pyrrolizidine alkaloids and their N-oxides in honey: Application to Echium vulgare honeys. J Agric Food Chem 2005; 53: $1894-902$

112 Ioset JR, Raoelison GE, Hostettmann K. Detection of aristolochic acid in Chinese phytomedicines and dietary supplements used as slimming regimens. Food Chem Toxicol 2003; 41: 29-36

113 Koh HL, Wang H, Zhou S, Chan E, Woo SO. Detection of aristolochic acid I, tetrandrine and fangchinoline in medicinal plants by high performance liquid chromatography and liquid chromatography/mass spectrometry. J Pharm Biomed Anal 2006; 40: 653-61

114 Careri M, Mangia A, Musci M. Overview of the applications of liquid chromatography mass spectrometry interfacing systems in food analysis: naturally occurring substances in food. J Chromatogr A 1998; 794: $263-97$

115 Sashidhara $K V$, Rosaiah JN. Various dereplication strategies using LCMS for rapid natural product lead identification and drug discovery. Nat Prod Commun 2007; 2: 193-202

116 Xing J, Xie CF, Lou HX. Recent applications of liquid chromatographymass spectrometry in natural products bioanalysis. J Pharm Biomed Anal 2007; 44: $368-78$

117 Wolfender JL, Ndjoko K, Hostettmann K. The potential of LC-NMR in phytochemical analysis. Phytochem Anal 2001; 12: 2-22

118 Duckett CJ, Lindon JC, Walker H, Abou-Shakra F, Wilson ID, Nicholson $J K$. Metabolism of 3-chloro-4-fluoroaniline in rat using [C-14]-radiolabelling, F-19-NMR spectroscopy, HPLC-MS/MS, HPLC-ICPMS and HPLC-NMR. Xenobiotica 2006; 36: 59-77
119 Wolfender JL, Queiroz EF, Hostettmann K. The importance of hyphenated techniques in the discovery of new lead compounds from nature. Exp Opin Drug Discov 2006; 1: 237-60

120 Waridel P, Wolfender JL, Ndjoko K, Hobby KR, Major HJ, Hostettmann $K$. Evaluation of quadrupole time-of-flight tandem mass spectrometry and ion-trap multiple-stage mass spectrometry for the differentiation of C-glycosidic flavonoid isomers. J Chromatogr A 2001; 926: $29-41$

121 Wolfender JL, Queiroz EF, Hostettmann K. Development and application of LC/NMR techniques to the identification of bioactive natural products. In: Colegate SM, Molyneux RJ, editors. Bioactive natural products; detection, isolation and structural determination. London: CRC press; 2008: $143-90$

122 Wilson ID, Brinkman UAT. Hype and hypernation: multiple hyphenation of column liquid chromatography and spectroscopy. Trends Anal Chem 2007; 26: 847 - 54

123 Hall C. Dictionary of natural products on CD-ROM: Chapman \& Hall; 2008

124 Jaroszewski JW. Hyphenated NMR methods in natural products research, Part 1: Direct hyphenation. Planta Med 2005; 71: 691-700

125 Jaroszewski JW. Hyphenated NMR methods in natural products research, Part 2: HPLC-SPE-NMR and other new trends in NMR hyphenation. Planta Med 2005; 71: $795-802$

126 Clarkson C, Madikane EV, Hansen SH, Smith PJ, Jaroszewski JW. HPLCSPE-NMR characterization of sesquiterpenes in an antimycobacterial fraction from Warburgia salutaris. Planta Med 2007; 73: 578-84

127 Lambert M, Wolfender JL, Staerk D, Christensen B, Hostettmann K, Jaroszewski JW. Identification of natural products using HPLC-SPE combined with CapNMR. Anal Chem 2007; 79: 727-35

128 Louden D, Handley A, Taylor S, Lenz E, Miller S, Wilson ID et al. Spectroscopic characterization and identification of ecdysteroids using high-performance liquid chromatography combined with on-line UV-diode array, FT-infrared and $1 \mathrm{H}$-nuclear magnetic resonance spectroscopy and time of flight mass spectrometry. J Chromatogr A 2001; 910: $237-46$

129 Ren DM, Guo HF, Wang SQ Lou HX. Separation and structure determination of two diastereomeric pairs of enantiomers from Dracocephalum rupestre by high-performance liquid chromatography with circular dichroism detection. J Chromatogr A 2007; 1161: 334 - 7

130 Huber L, George SA. Diode array detection in HPLC. New York: Marcel Dekker; 1993

131 Larsen TO, Hansen MAE. Dereplication and discovery of natural products by UV spectroscopy. In: Colegate SM, Molyneux RJ, editors. Bioactive natural products: detection, isolation, and structural determination, 2nd Edition London: CRC Press; 2008: 221 - 44

132 Robbins RJ. Phenolic acids in foods: An overview of analytical methodology. J Agric Food Chem 2003; 51: 2866-87

133 Waridel $P$, Wolfender JL, Lachavanne JB, Hostettmann K. Identification of the polar constituents of Potamogeton species by HPLC-UV with postcolumn derivatization, HPLC-MS(n) and HPLC-NMR, and isolation of a new ent-labdane diglycoside. Phytochemistry 2004; 65: 2401 - 10

134 Bennett RN, Mellon FA, Foidl N, Pratt JH, Dupont MS, Perkins L et al. Profiling glucosinolates and phenolics in vegetative and reproductive tissues of the multi-purpose trees Moringa oleifera L. (horseradish tree) and Moringa stenopetala L. J Agric Food Chem 2003; 51: $3546-53$

135 Gobbo-Neto L, Lopes NP. Online identification of chlorogenic acids, sesquiterpene lactones, and flavonoids in the Brazilian arnica Lychnophora ericoides Mart. (Asteraceae) leaves by HPLC-DAD-MS and HPLC-DAD-MS/MS and a validated HPLC-DAD method for their simultaneous analysis. J Agric Food Chem 2008; 56: 1193 - 204

136 Yang M, Sun J, Lu Z, Chen G, Guan S, Liu X et al. Phytochemical analysis of herbal medicines using liquid chromatography coupled with mass spectrometry. J Chromatogr A; 2008: doi:10.1016/j.chroma.2008.08.097

137 Kussmann M, Affolter M, Nagy K, Holst B, Fay LB. Mass Spectrometry in nutrition: Understanding dietary health effects at the molecular level. Mass Spectrom Rev 2007; 26: 727-50

138 Wolfender JL, Rodriguez S, Hostettmann K. Liquid chromatography coupled to mass spectrometry and nuclear magnetic resonance spectroscopy for the screening of plant constituents. J Chromatogr A 1998; 794: 299-316

139 Suzuki H, Sasaki R, Ogata Y, Nakamura Y, Sakurai N, Kitajima M et al. Metabolic profiling of flavonoids in Lotus japonicus using liquid chromatography Fourier transform ion cyclotron resonance mass spectrometry. Phytochemistry 2008; 69: 99-111 
140 Bonkanka CX, Smelcerovic A, Zuehlke S, Rabanal RM, Spiteller M, Sanchez-Mateo CD. HPLC-MS analysis and anti-oedematogenic activity of Hypericum grandifolium choisy (Hypericaceae). Planta Med 2008; 74: 719-25

141 Han J, Ye M, Xu M, Qiao X, Chen HL, Wang BR et al. Comparison of phenolic compounds of rhubarbs in the section deserticola with Rheum palmatum by HPLC-DAD-ESI-MSn. Planta Med 2008; 74: 873 - 9

142 Hopfgartner G. Identification of metabolites by multi-instruments strategies (LC-MS-MS on various platforms). In: Niessen WMA, editor. Encyclopedia of mass spectrometry,Vol 8: Hyphenated methods. Oxford: Elsevier Science; 2006: 465 - 74

143 Sun F, He Q Shi PY, Xiao PG, Cheng YY. Characterization and identification of triterpenoid saponins in crude extracts from Clematis spp. by high-performance liquid chromatography/electrospray ionization with multi-stage tandem mass spectrometry. Rapid Commun Mass Spectrom 2007; 21: $3743-50$

144 Fuzzati N, Gabetta N, Strepponi I, Villa F. High-performance liquid chromatography-electrospray ionization mass spectrometry and multiple mass spectrometry studies of hyperforin degradation products. J Chromatogr A 2001; 926: 187-98

145 Cuyckens F, Claeys M. Mass spectrometry in the structural analysis of flavonoids. J Mass Spectrom 2004; 39: 1 - 15

146 Kuhn F, Oehme M, Romero F, Abou-Mansour E, Tabacchi R. Differentiation of isomeric flavone/isoflavone aglycones by MS2 ion trap mass spectrometry and a double neutral loss of CO. Rapid Commun Mass Spectrom 2003; 17: 1941 -9

147 Biemann K. Sequencing of peptides by tandem mass spectrometry and high-energy collision induced dissociation. Methods Enzymol 1990; 193: $455-79$

148 De Abreu MB, Dal Piaz F, Temraz A, Bader A, De Tommasi N, Braca A. Gypsins A-D from Gypsophila arabica. J Nat Prod 2008; 71: 1336 - 42

149 Wolff JC, Eckers C, Sage AB, Giles K, Bateman R. Accurate mass liquid chromatography/mass spectrometry on quadrupole orthogonal acceleration time-of flight mass analyzers using switching between separate sample and reference sprays. 2. Applications using the dual-electrospray ion source. Anal Chem 2001; 73: 2605-12

150 Wolfender JL, Queiroz EF, Hostettmann K. Phytochemistry in the microgram domain - a LC-NMR perspective. Magn Reson Chem 2005; 43: 697 - 709

151 Yang Z. Online hyphenated liquid chromatography-nuclear magnetic resonance spectroscopy for drug metabolite and nature product analysis. Pharm Biomed Anal 2006; 40: 516-27
152 Cogne AL, Queiroz EF, Marston A, Wolfender JL, Mavi S, Hostettmann K. On-line identification of unstable iridoids from Jamesbrittenia fodina by HPLC-MS and HPLC-NMR. Phytochem Anal 2005; 16: 429-39

153 Exarchou V, Krucker M, van Beek TA, Vervoort J, Gerothanassis IP, Albert K. LC-NMR coupling technology: recent advancements and applications in natural products analysis. Magn Reson Chem 2005; 43: $681-7$

154 Olson DL, Norcross JA, O'Neil-Johnson M, Molitor PF, Detlefsen DJ, Wilson AG et al. Microflow NMR: concepts and capabilities. Anal Chem 2004; 76: 2966 - 74

155 Hu JF, Garo E, Yoo HD, Cremin PA, Zeng L, Goering MG et al. Application of capillary-scale NMR for the structure determination of phytochemicals. Phytochem Anal 2005; 16: 127-33

156 Glauser G, Guillarme D, Grata E, Boccard J, Thiocone A, Carrupt PA et al. Optimized liquid chromatography-mass spectrometry approach for the isolation of minor stress biomarkers in plant extracts and their identification by capillary nuclear magnetic resonance. J Chromatogr A 2008; 1180: $90-8$

157 Seger C, Sturm S. Analytical aspects of plant metabolite profiling platforms: Current standings and future aims. J Proteome Res 2007; 6: $480-97$

158 Choi YH, Choi HK, Peltenburg-Looman AMG, Lefeberz AWM, Verpoorte $R$. Quantitative analysis of ginkgolic acids from Ginkgo leaves and products using H-1-NMR. Phytochem Anal 2004; 15: 325 - 30

159 Tatsis EC, Exarchou V, Troganis AN, Gerothanassis IP. H-1 NMR determination of hypericin and pseudohypericin in complex natural mixtures by the use of strongly deshielded $\mathrm{OH}$ groups. Anal Chim Acta 2008; 607: 219-26

160 Huck CW, Abel G, Popp M, Bonn GK. Comparative analysis of naphthodianthrone and phloroglucine derivatives in St. John's Wort extracts by near infrared spectroscopy, high-performance liquid chromatography and capillary electrophoresis. Anal Chim Acta 2006; 580: $223-30$

161 Yang SY, Kim HK, Lefeber AWM, Erkelens C, Angelova N, Choi YH et al. Application of two-dimensional nuclear magnetic resonance spectroscopy to quality control of ginseng commercial products. Planta Med 2006; 72: 364-9

162 Cardoso-Taketa AT, Pereda-Miranda R, Choi YH, Verpoorte R, Villarreal $M L$. Metabolic profiling of the Mexican anxiolytic and sedative plant Golphimia glauca using nuclear magnetic resonance spectroscopy and multivariate data analysis. Planta Med 2008; 74: 1295 - 301

163 Potterat 0 , Hamburger $M$. Natural products in drug discovery - Concepts and approaches for tracking bioactivity. Curr Org Chem 2006; 10: $899-920$ 